\title{
A PHOTOMETRIC AND SPECTROSCOPIC STUDY OF DWARF AND GIANT GALAXIES IN THE COMA CLUSTER. IV. THE LUMINOSITY FUNCTION ${ }^{1}$
}

\author{
Bahram Mobasher, ${ }^{2,3}$ Matthew Colless, ${ }^{4}$ Dave Carter,${ }^{5}$ Bianca M. Poggianti, ${ }^{6}$ Terry J. Bridges, ${ }^{7}$ Kelly Kranz, ${ }^{4}$ \\ Y. Komiyama, ${ }^{8}$ N. Kashikawa, ${ }^{9}$ M. Yagi, ${ }^{9}$ and S. OKamura ${ }^{10}$ \\ Received 2002 September 16; accepted 2002 December 26
}

\begin{abstract}
A large spectroscopic survey is constructed of galaxies in the Coma Cluster. The survey covers a wide area $\left(1 \mathrm{deg}^{2}\right)$ to deep magnitudes $(R \sim 19.5)$, covering both the core (high density) and outskirts (intermediate to low density) of the cluster. The spectroscopic sample consists of a total of 1191 galaxies, of which 760 are confirmed members of the Coma Cluster. A statistical technique is developed to correct the spectroscopic sample for incompleteness. The corrected sample is then used to construct an $R$-band luminosity function (LF) spanning a range of $7 \mathrm{mag}\left(-23<M_{R}-5 \log h_{65}<-16\right)$ at both the core and outskirts of the cluster. The $R$-band LF for the entire Coma Cluster, fitted to Schechter form, gives $M_{R}^{*}=-21.79_{-0.09}^{+0.08}+5 \log h_{65}$ and $\alpha=-1.18_{-0.02}^{+0.04}$. Dependence of the LF on local environment in Coma is explored. The LFs are found to be the same, within the errors, between the inner and outer regions and close to those from recent measurements for field galaxies. This is remarkable given the variation in the spectral types of galaxies between field and cluster environments. The steep faint-end slope for the LFs, observed in previous studies using photometric surveys, is not found here. However, the $L F$ in this study is only measured to $M_{R}=-16$, compared to much deeper limits $\left(M_{R} \sim-12\right)$ achieved in photometric surveys. The total $B$-band LF for the Coma Cluster, fitted to a Schechter form, is $M_{B}^{*}=-19.95_{-0.10}^{+0.15}+5 \log h_{65}$ and $\alpha=-0.96_{-0.02}^{+0.01}$. This also shows a dip at $M_{B}^{*}=-18 \mathrm{mag}$, in agreement with previous studies. The implications of this feature are discussed. The LF is studied in $B-R$ color intervals and shows a steep faint-end slope for red $(B-R>1.35)$ galaxies, at both the core and outskirts of the cluster. This population of low-luminosity red galaxies has a higher surface density than the blue $(B-R<1.35)$ star-forming population and dominates the faint end of the Coma Cluster LF. It is found that the relative number of high surface brightness galaxies is larger at the cluster core, implying the destruction of low surface brightness galaxies in the dense core environment.
\end{abstract}

Subject headings: galaxies: clusters: general — galaxies: clusters: individual (Coma) — galaxies: distances and redshifts — galaxies: kinematics and dynamics — galaxies: luminosity function, mass function

\section{INTRODUCTION}

Detailed knowledge of the luminosity function (LF), defined as the number density of galaxies with a given luminosity, is essential for any observational study of the formation and evolution of galaxies and for constraining galaxy formation scenarios and models for large-scale structure. Over the last three decades, extensive studies of LFs have

\footnotetext{
${ }^{1}$ Based on observations made with the William Herschel Telescope operated on the island of La Palma by the Isaac Newton Group in the Spanish Observatorio del Roque de los Muchachos of the Instituto de Astrofísica de Canarias, and also the Anglo-Australian Telescope of the Anglo-Australian Observatory.

${ }^{2}$ Space Telescope Science Institute, 3700 San Martin Drive, Baltimore, MD 21218.

${ }^{3}$ Affiliated with the Space Telescope Division of the European Space Agency.

${ }^{4}$ Research School of Astronomy and Astrophysics, Australian National University, Weston Creek, ACT 2611, Australia.

${ }^{5}$ Astrophysics Research Institute, Liverpool John Moores University, Twelve Quays House, Egerton Wharf, Birkenhead, Wirral, CH41 1LD, UK.

${ }^{6}$ Osservatorio Astronomico di Padova, vicolo dell'Osservatorio 5, 35122 Padua, Italy.

${ }^{7}$ Anglo-Australian Observatory, P.O. Box 296, Epping, NSW 2121, Australia.

${ }^{8}$ Subaru Telescope, 650 North A'ohoku Place, Hilo, HI 96720

${ }^{9}$ National Astronomical Observatory, Mitaka, Tokyo 181-8588, Japan.

${ }^{10}$ Department of Astronomy, University of Tokyo, Bunkyo-ku, Tokyo 113-0033, Japan.
}

been performed, in both clusters and general fields. Although the move from wide-area photographic plate surveys to high-performance, sensitive CCDs has greatly advanced the subject, there are still a number of questions that remain unanswered, including the following:

1. Is a single parametric form for the LF a reasonable approximation over the entire luminosity range?

2. What is the dependence of the LF on environment, and does this also depend on the selection characteristics of the sample (e.g., depth, completeness)?

3. How do the LFs for different Hubble types or color intervals compare?

4. Do the high and low surface brightness galaxies follow different LFs?

5. How do the LFs of giant and dwarf galaxies compare?

To address these questions, one needs a wide-area survey, complete to deep magnitudes. Moreover, in comparing different LFs, one must minimize observational biases (e.g., different depths) and selection effects (e.g., incompleteness). These are some of the reasons for slow progress in this field.

Study of the field LF requires a redshift survey, complete to some magnitude limit. Because of the small numbers of intrinsically faint galaxies in even the largest magnitudelimited redshift surveys, field LFs do not normally extend to faint magnitudes. While this can be avoided for cluster galaxies, since all the objects are roughly at the same distance, there are still problems at faint magnitudes due to 
contamination by background objects. Moreover, compared to isolated systems, cluster galaxies are subject to dynamical evolution, affecting the shape and characteristics of their LF. The former problem can be resolved by exploiting a deep spectroscopic sample in clusters (and so spectroscopically confirmed cluster members), while the latter is best addressed by studying large areas around clusters, covering both low- and high-density regions.

In this paper, we aim to explore the above questions by constructing the LF in the Coma Cluster, avoiding potential problems that have affected previous studies. This has become possible with the advent of large-format CCDs, allowing wide-area surveys to deep magnitudes. Combining these with fiber-fed spectrographs, one could then construct statistical samples of spectroscopic data on galaxies to faint magnitudes.

At a redshift of $z=0.023$, Coma is the richest nearby cluster, providing a laboratory for studying evolution of different types of galaxies. Other nearby rich clusters, such as Virgo and Ursa Major, are relatively unevolved, not allowing a direct comparison of the properties of galaxies as a function of their local environment. A detailed study of Coma also provides a control sample to compare with more distant clusters. This improves previous works in many aspects, including the following:

1. wide-area CCD coverage of the cluster, extending more than $1^{\circ}$ from its center (this also covers the NGC 4839 group);

2. full spectroscopic information for galaxies in the sample, allowing a measure of the LF independent of uncertain background corrections;

3 . inclusion of the faintest galaxies for which spectroscopic data can be obtained $(R \sim 19.5)$, allowing strong constraints on the shape of the LF at faint magnitudes;

4. availability of accurate CCD photometry in both the $B$ and $R$ bands, providing color information for galaxies in the sample;

5. a well-defined selection function for the spectroscopic sample.

This is the fourth paper in a series studying the nature of giant and dwarf galaxies in the Coma Cluster, using photometric (Komiyama et al. 2002, hereafter Paper I) and spectroscopic (Mobasher et al. 2001, hereafter Paper II) observations. Analyses of the diagnostic line indices (Poggianti et al. 2001, hereafter Paper III), the radial dependence of galaxy properties (Carter et al. 2002, hereafter Paper V), and the dynamics of giant and dwarf populations (Edwards et al. 2002) have already been performed. Future papers will present a spectroscopic comparison with intermediate redshift clusters (B. M. Pogianti et al. 2003, in preparation) and a study of the scatter and environmental dependence of the color-magnitude relation (B. Mobasher et al. 2003, in preparation).

In the next section, observations and spectroscopic sample selection are discussed. The selection functions are derived in $\S 3$. Section 4 presents the LFs and their dependence on different physical parameters. The results are then compared with other studies in $\S 5$, followed by a discussion of the results in $\S 6$. The conclusions are listed in $\S 7$. We assume a distance modulus of $\mathrm{DM}=35.13 \mathrm{mag}$ for the Coma Cluster, corresponding to $H_{0}=65 \mathrm{~km} \mathrm{~s}^{-1} \mathrm{Mpc}^{-1}$.

\section{OBSERVATIONS AND SAMPLE SELECTION}

To address the aims of this study, photometric and spectroscopic observations were designed to survey a wide area of the cluster (consisting of both core and outskirt fields) to deep flux levels. This is then complemented by a sample of brighter galaxies with spectroscopic data, compiled from different sources. The result is a sufficiently large spectroscopic sample to allow a detailed study of the LF of galaxies. The procedure for selecting the spectroscopic sample and its properties is summarized as follows.

A wide-area photometric survey was carried out by the MCCD (a mosaic camera consisting of $5 \times 8 \mathrm{CCDs}$ and a field of view of $0.5 \mathrm{deg}^{2}$; Sekiguchi et al. 1998) on the William Herschel Telescope (WHT). A total of five fields in the Coma Cluster were surveyed, each $32.5 \times 50.8 \mathrm{arcmin}^{2}$. The photometric surveys were performed in both the $B$ and $R$ bands and are complete to $R=21$. Details of the photometric observations, data reductions, source extraction, and the construction of the photometric catalogs are given in Paper I. Follow-up medium-resolution (6-9 A) spectroscopic observations, using WYFFOS on the WHT, were then performed on two fields of this survey: Coma 1 (at the center) and Coma 3 (southwest of Coma 1, including the NGC 4839 group). The spectroscopic sample has a magnitude limit of $R=19.75$, with the spectra having large enough signal-to-noise ratios to allow accurate measurement of diagnostic line indices (Paper III). The coordinates of the center of fields and the total number of objects in the spectroscopic sample in each field are listed in Table 1. The spectroscopic sample selection, spectroscopic observations, and data reduction are explained in detail in Paper II, where the spectroscopic catalog is also presented. The criteria for selecting the spectroscopic sample in the central (Coma 1) and outskirt (Coma 3 ) fields are the same, with no radially dependent selection biases present. This sample will be referred to as the Deep Spectroscopic Survey (DSS).

TABLE 1

Coordinates of the Center of Each Field and the Number of Sources with Spectroscopic Data In the SSS and DSS Samples

\begin{tabular}{|c|c|c|c|c|c|c|}
\hline \multirow[b]{2}{*}{ FIELD } & \multirow[b]{2}{*}{ R.A. (J2000.0) } & \multirow[b]{2}{*}{ DECL. (J2000.0) } & \multicolumn{2}{|c|}{ DSS } & \multicolumn{2}{|c|}{ SSS } \\
\hline & & & Members & Total & Members & Total \\
\hline Coma 1 .................. & 125923.7 & 280112.5 & 189 & 302 & 282 & 369 \\
\hline Coma 2 ................ & 125707.5 & 280112.5 & $\ldots$ & $\ldots$ & 97 & 184 \\
\hline Coma 3. & 125707.5 & 271113.0 & 90 & 188 & 102 & 148 \\
\hline
\end{tabular}

Note.-Units of right ascension are hours, minutes, and seconds, and units of declination are degrees, arcminutes, and arcseconds. 
The DSS is complemented by another sample with a brighter spectroscopic magnitude limit $(R \sim 18)$, based on the compilation from Colless \& Dunn (1996), and another spectroscopic survey of Coma galaxies, carried out using the Two-Degree Field (2dF) spectroscopic facility at the Anglo-Australian Telescope (Edwards et al. 2002). This sample is referred to as the Shallow Spectroscopic Survey (SSS). The total number of galaxies in each field for the SSS sample is also listed in Table 1. Line indices are not available for the SSS sample.

The spatial distribution of galaxies in the DSS and SSS is shown in Figure 1, with the location and size of the MCCD survey areas overlaid: the Coma 1 field at the center of the cluster, the Coma 2 field to the west of Coma 1, and the Coma 3 field to the southwest of Coma 1. Comparison between the redshifts of galaxies common to both the DSS and SSS samples shows a mean difference of $28 \pm 9 \mathrm{~km} \mathrm{~s}^{-1}$ between the estimated velocities in the two surveys.

The final spectroscopic catalog is compiled by combining galaxies with available redshifts from the DSS and SSS samples, the $B$ - and $R$-band CCD data (i.e., magnitudes and surface brightness values) from the MCCD photometric survey, and the line indices for galaxies in the Coma 1 and Coma 3 fields. For galaxies common to both spectroscopic surveys, the mean redshifts were calculated. The $B$ - and $R$-band magnitudes in this study are measured over a circular aperture of radius 3 times the Kron radius (see Paper II). This has the advantage of scaling the photometric aperture in proportion to the size of galaxies and is therefore less susceptible to underestimating the magnitudes for larger galaxies, as is often the case if a constant aperture is used for all the objects at the same distance. The Kron magnitudes used here are close to total (see Paper I for details). The $B-R$ colors are also measured over circular apertures of 3 times the Kron radius and, hence, correspond closely to total colors.

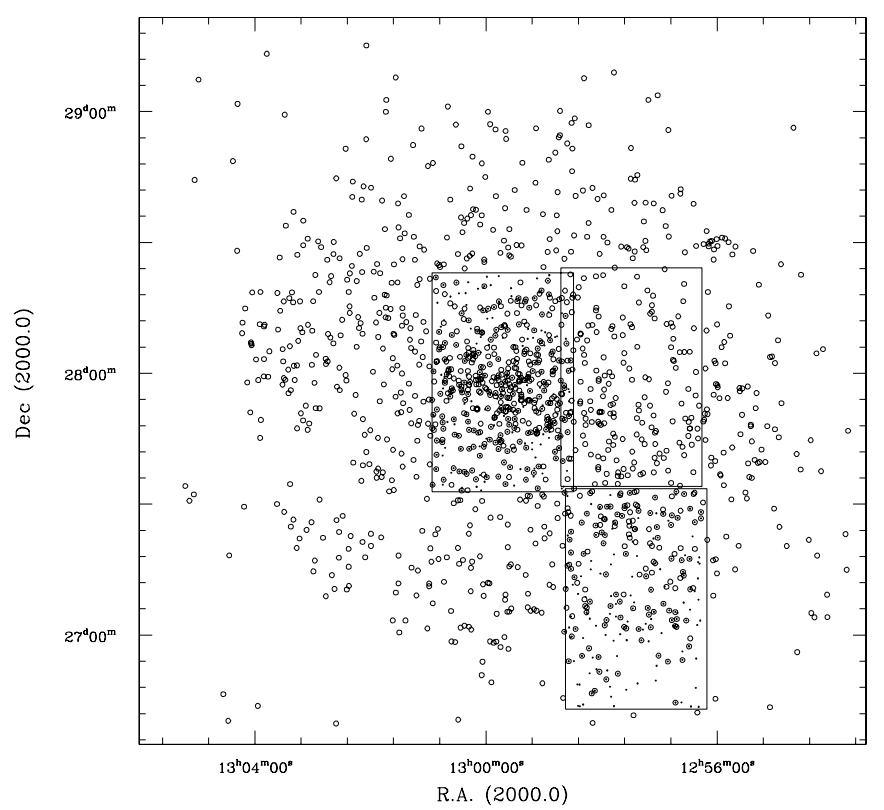

FIG. 1.-Distribution of galaxies with spectroscopic data from DSS (filled circles), SSS (open circles), and galaxies common to both surveys (dotted circles). The three fields with available spectroscopic data are shown as rectangles: Coma 1 (central), Coma 2 (west of Coma 1), and Coma 3 (southwest of Coma 1). The total number of galaxies and the coordinates of the center of each field are listed in Table 1.
Galaxies in the range $4000 \mathrm{~km} \mathrm{~s}^{-1}<c z<10,000 \mathrm{~km} \mathrm{~s}^{-1}$ are considered to be members of the Coma Cluster (Colless \& Dunn 1996). The total number of galaxies identified as cluster members in each of the three fields here is also listed in Table 1. In the case of the Coma 2 field, only spectroscopic data from the SSS are available. For this reason, the depth of the spectroscopic survey in this field is shallower than that in the other two fields, with a different completeness function, as discussed in the next section.

The photometric properties of galaxies in the spectroscopic sample are explored using their $B-R$ colors and $R$ band effective surface brightness $\left(\mu_{\mathrm{eff}}\right)$ distributions in Figures 2 and 3, respectively. The effective surface brightness is defined as the mean surface brightness within the effective radius (radius containing half the total light of the galaxy, with the latter assumed to be the luminosity inside an aperture 3 times the Kron radius of that galaxy; Paper I) of the galaxy. The effect of seeing on the observed surface brightness was considered and found to be negligible.

There are four galaxies with $B-R>2$ that are confirmed members of the Coma Cluster (two galaxies in Coma 1 and one galaxy in each of the Coma 2 and Coma 3 fields). Such red galaxies were previously considered to be background objects (Conselice et al. 2002), with none so far identified as a Coma Cluster member. Considering the surface brightness distributions, we find that cluster members have a relatively brighter effective surface brightness compared to nonmembers (when corrected for surface brightness dimming and the $k$-correction due to redshift), with the tail of the surface brightness distribution for cluster members extending to brighter values. The median effective surface brightness changes from $\mu_{\mathrm{eff}}=20.50 \mathrm{mag} \operatorname{arcsec}^{-2}$ in the cluster to $21.50 \mathrm{mag} \operatorname{arcsec}^{-2}$ for the field galaxies. While this was known from previous studies, there is also evidence for a monotonic decrease in surface brightness from the core (Coma 1) to the outskirts (Coma 3).

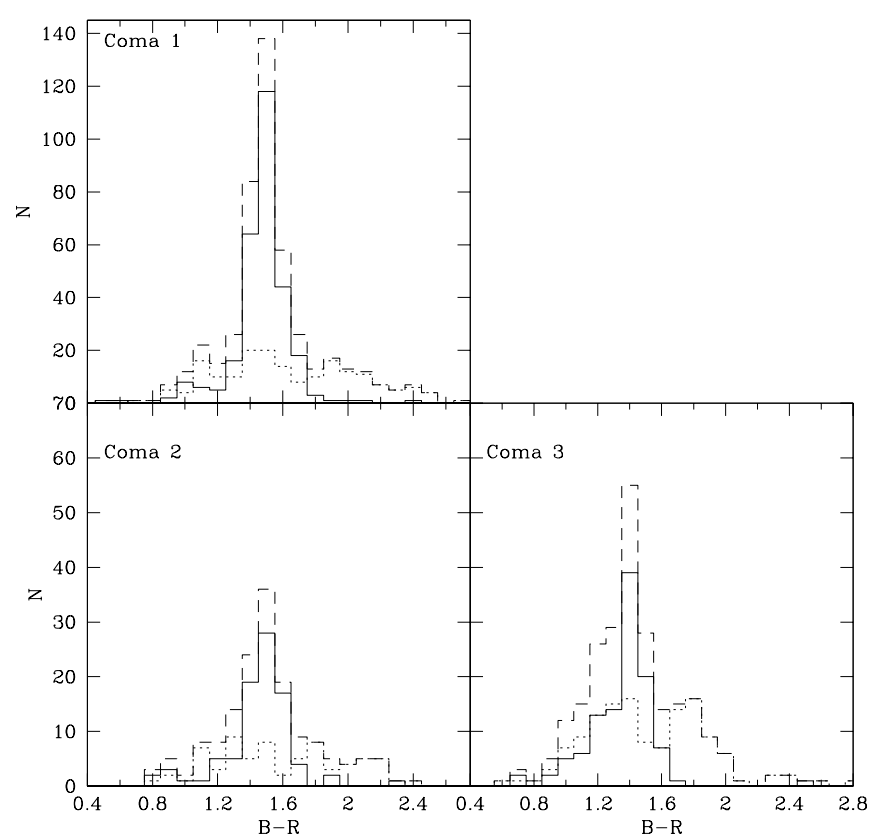

FIG. 2.- $-B-R$ color distributions in the three fields for the entire DSS+SSS spectroscopic sample (dashed line), for spectroscopically confirmed cluster members (solid line), and for nonmembers (dotted line). 


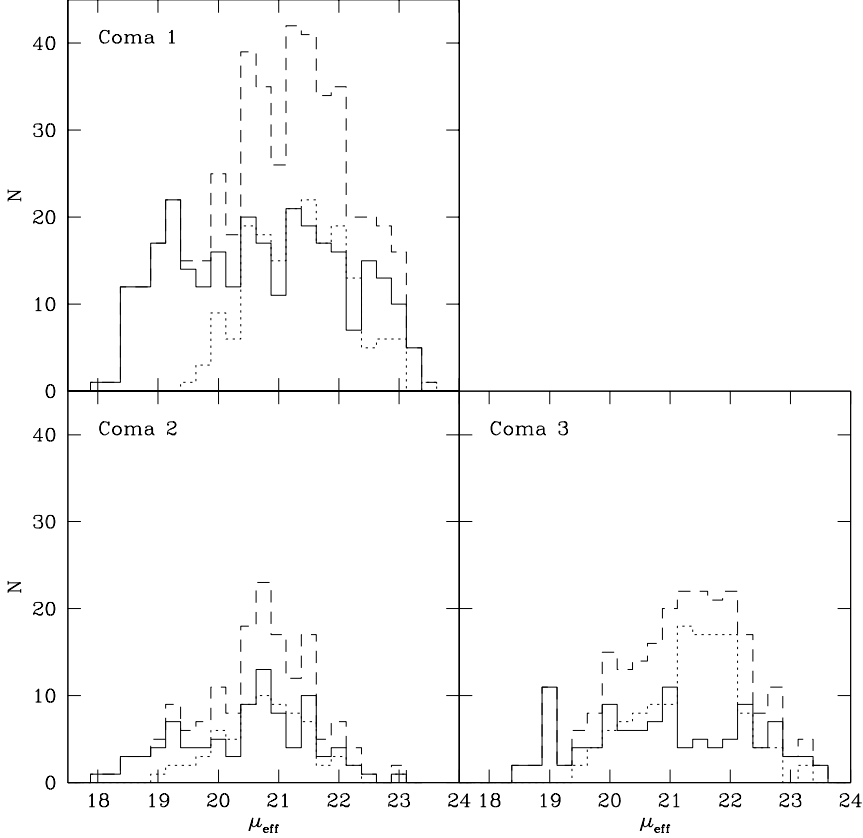

FIG. 3.- $R$-band surface brightness distributions in the three fields for the entire DSS+SSS spectroscopic sample (dashed line), for spectroscopically confirmed cluster members (solid line), and for nonmembers (dotted line)

\section{CLUSTER MEMBERSHIP}

The spectroscopic surveys are incomplete, so we do not know for every individual galaxy whether or not it is a member of the cluster. Therefore, in order to compute the cluster LF, we assume that the spectroscopic sample is representative, in the sense that the fraction of galaxies that are cluster members (which depends on both magnitude and position) is the same in the (incomplete) spectroscopic sample as in the (complete) photometric sample. If this assumption applies to both the spectroscopic surveys we are using, then they can be straightforwardly combined to give the cluster membership fraction over the full magnitude range of the two surveys.

If $N_{c}(m, r \mid \mathrm{spec})$ and $N_{t}(m, r \mid \mathrm{spec})$ are, respectively, the number of cluster members (based on their redshifts) and the total number of galaxies within the combined spectroscopic sample in given ranges of apparent magnitude and cluster radius, then the probability of a randomly selected galaxy in the combined spectroscopic sample being a member of the Coma Cluster, $P(m, r \mid$ spec $)$, is

$$
P(m, r \mid \mathrm{spec})=\frac{N_{c}(m, r \mid \mathrm{spec})}{N_{t}(m, r \mid \mathrm{spec})} .
$$

We simplify this by taking as our radial bins the ranges covered by the three fields of the survey, so that we have

$$
P_{i}(m \mid \mathrm{spec})=\frac{N_{c, i}(m \mid \mathrm{spec})}{N_{t, i}(m \mid \mathrm{spec})},
$$

where $i=1,2,3$ corresponds to the Coma 1, Coma 2, and Coma 3 fields.

The cluster membership fractions as functions of apparent magnitude are presented in Figure 4 and listed in Table 2 for each of the three fields. The uncertainties in

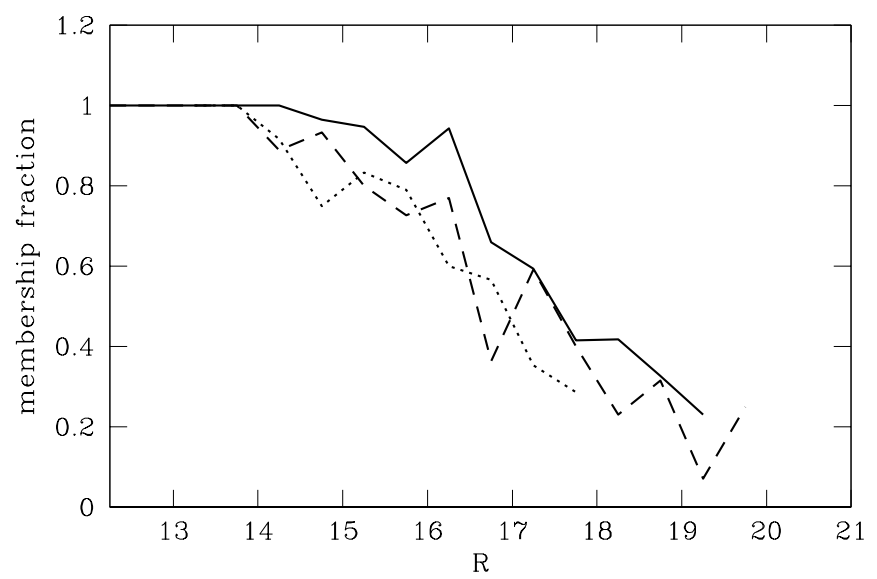

FIG. 4.-Cluster membership fractions as a function of apparent magnitude for Coma 1 (solid line), Coma 2 (dotted line), and Coma 3 (dashed line).

these fractions, assuming Poisson statistics, are estimated as

$$
\frac{d P_{i}(m \mid \mathrm{spec})}{P_{i}(m \mid \mathrm{spec})}=\left[\frac{1}{N_{c, i}(m \mid \mathrm{spec})}-\frac{1}{N_{t, i}(m \mid \mathrm{spec})}\right]^{1 / 2},
$$

where $N_{c, i}(m \mid \mathrm{spec})$ is a binomial variable with its corresponding variance, as discussed in the next section. The errors corresponding to each magnitude interval in each field are also listed in Table 2. The membership fraction is determined down to $R \sim 19.5$ for the Coma 1 and Coma 3 fields, but only down to $R \sim 17.75$ for Coma 2 (since only the SSS is available in this field). As expected, the Coma 1 field, being centrally located, has a higher membership fraction at all magnitudes than the Coma 2 and Coma 3 fields. Coma 3 has a comparable membership fraction to Coma 2 at all magnitudes, even though it is farther from the center of the cluster; this reflects the presence of the NGC 4839 group in this field.

TABLE 2

Membership Fractions in $R$-Band Magnitude Intervals FOR THE THREE FIELDS

\begin{tabular}{cccc}
\hline \hline$R$ & Coma 1 & Coma 2 & Coma 3 \\
\hline $12.75 \ldots \ldots \ldots \ldots$. & $1.00(0.58)$ & $\ldots$ & $1.00(0.71)$ \\
$13.25 \ldots \ldots \ldots \ldots$. & $1.00(0.27)$ & $1.00(0.58)$ & $1.00(1.00)$ \\
$13.75 \ldots \ldots \ldots \ldots$. & $1.00(0.27)$ & $1.00(0.45)$ & $1.00(0.44)$ \\
$14.25 \ldots \ldots \ldots \ldots$. & $1.00(0.20)$ & $0.92(0.28)$ & $0.89(0.31)$ \\
$14.75 \ldots \ldots \ldots \ldots$. & $0.96(0.18)$ & $0.75(0.43)$ & $0.93(0.25)$ \\
$15.25 \ldots \ldots \ldots \ldots$. & $0.95(0.22)$ & $0.83(0.26)$ & $0.80(0.40)$ \\
$15.75 \ldots \ldots \ldots \ldots$. & $0.86(0.17)$ & $0.79(0.20)$ & $0.73(0.26)$ \\
$16.25 \ldots \ldots \ldots \ldots$. & $0.94(0.16)$ & $0.60(0.17)$ & $0.77(0.24)$ \\
$16.75 \ldots \ldots \ldots \ldots$. & $0.66(0.11)$ & $0.57(0.14)$ & $0.36(0.13)$ \\
$17.25 \ldots \ldots \ldots \ldots$. & $0.59(0.01)$ & $0.35(0.01)$ & $0.59(0.16)$ \\
$17.75 \ldots \ldots \ldots \ldots$. & $0.42(0.08)$ & $0.29(0.01)$ & $0.40(0.01)$ \\
$18.25 \ldots \ldots \ldots \ldots$. & $0.42(0.09)$ & $\ldots$ & $0.23(0.09)$ \\
$18.75 \ldots \ldots \ldots \ldots$. & $0.33(0.08)$ & $\ldots$ & $0.32(0.09)$ \\
$19.25 \ldots \ldots \ldots \ldots \ldots$. & $0.23(0.09)$ & $\ldots$ & $0.07(0.05)$ \\
$19.75 \ldots \ldots \ldots \ldots .$. & $\ldots$ & $\ldots$ & $0.25(0.25)$ \\
\hline
\end{tabular}

Note.-Errors are given in parentheses. Photometric errors $(0.03 \mathrm{mag})$ are significantly smaller than the bin sizes. 


\section{THE CLUSTER LUMINOSITY FUNCTION}

Let $N_{p, i}(m)$ be the number of galaxies as a function of magnitude $m$ and field $i$, in the full photometric sample. The cluster LF can then be estimated as

$$
\phi_{i}(m)=\frac{N_{p, i}(m)}{A_{i}} f_{i}(m),
$$

where $A_{i}$ is the area of each field, $N_{p, i}(m) / A_{i}$ the surface density in the photometric catalog, and $f_{i}(m)=P_{i}(m \mid \mathrm{spec})$ the cluster membership fraction.

The errors in the LF can be estimated by noting that $\phi_{i}(m)$ is the product of two random variables: $N_{p, i}(m)$, which is a Poisson random variable, and $N_{c, i}(m \mid \mathrm{spec})$, which is a binomial random variable [since it is the number of cluster members found in a fixed number of trials, $N_{t, i}(m \mid \mathrm{spec})$, where the probability of a single trial yielding a cluster member is the true cluster membership fraction, $\left.f_{i}(m)\right]$. The relative error in the LF is given by the quadrature sum of the relative errors in $N_{p, i}(m)$ and $N_{c, i}(m \mid \mathrm{spec})$, so that

$$
\frac{\operatorname{var}\left[\phi_{i}(m)\right]}{\phi_{i}(m)^{2}}=\frac{\operatorname{var}\left[N_{p, i}(m)\right]}{N_{p, i}(m)^{2}}+\frac{\operatorname{var}\left[N_{c, i}(m \mid \mathrm{spec})\right]}{N_{c, i}(m \mid \mathrm{spec})^{2}} .
$$

The variance in the Poisson variable is its expectation value, $\operatorname{var}\left[N_{p, i}(m)\right]=N_{p, i}(m)$, while the variance in the binomial variable is $\operatorname{var}\left[N_{c, i}(m \mid \mathrm{spec})\right]=N_{t, i}(m \mid \mathrm{spec}) f_{i}(m)\left[1-f_{i}(m)\right]$.
The relative error in the LF is thus

$$
\begin{aligned}
\frac{\delta \phi_{i}(m)}{\phi_{i}(m)} & \equiv \frac{\operatorname{var}\left[\phi_{i}(m)\right]^{1 / 2}}{\phi_{i}(m)} \\
& =\left[\frac{1}{N_{p, i}(m)}+\frac{1}{N_{c, i}(m \mid \mathrm{spec})}-\frac{1}{N_{t, i}(m \mid \mathrm{spec})}\right]^{1 / 2} .
\end{aligned}
$$

This expression reduces to $N_{c, i}(m \mid \mathrm{spec})^{-1 / 2}$ if $N_{t, i}(m \mid \mathrm{spec})=N_{p, i}(m)$ (i.e., if the spectroscopic sample is complete) and to $N_{p, i}(m)^{-1 / 2}$ if $N_{c, i}(m \mid \mathrm{spec})=N_{t, i}(m \mid \mathrm{spec})$ (i.e., if the membership fraction is unity).

The LFs for the Coma 1, Coma 2, and Coma 3 fields are listed in Table 3 and presented in Figure 5. Magnitudes are corrected for both Galactic absorption $\left(A_{R}=0.02\right)$ and redshift $\left(K_{z}=0.02\right)$ dimming. The distance of the Coma Cluster is then estimated as $m-M=\mathrm{DM}+A_{R}+K_{z}$, where $\mathrm{DM}=35.13 \mathrm{mag}$ is its distance modulus (corresponding to $H_{0}=65 \mathrm{~km} \mathrm{~s}^{-1} \mathrm{Mpc}^{-1}$ ). At this distance, $1^{\circ}=1.77 \mathrm{Mpc}$ (assuming $q_{0}=0.5$ ), corresponding to an area of $1.619 \mathrm{Mpc}^{2}$ for each of the three fields surveyed in this study. The LFs span the range $-22<M_{R}-5 \log h_{65}<-16$, except for the Coma 2 field, which is only based on the shallower SSS sample and, hence, only extends to $M_{R}=-18$.

It is clear from Figure 5 that the bright ends of the LFs are not well constrained from these three fields (filled

\begin{tabular}{|c|c|c|c|c|c|c|}
\hline \multirow[b]{2}{*}{$M_{R}$} & \multicolumn{2}{|c|}{ Сома 1} & \multicolumn{2}{|c|}{ Сома 2} & \multicolumn{2}{|c|}{ Сома 3} \\
\hline & $\phi_{1}(M)$ & $\Delta\left[\phi_{1}(M)\right]$ & $\phi_{2}(M)$ & $\Delta\left[\phi_{2}(M)\right]$ & $\phi_{3}(M)$ & $\Delta\left[\phi_{3}(M)\right]$ \\
\hline \multicolumn{7}{|c|}{ Extended SSS } \\
\hline$-23.00 \ldots \ldots \ldots \ldots \ldots$ & 2.16 & 1.53 & 0.72 & 0.51 & 0.72 & 0.51 \\
\hline$-22.75 \ldots \ldots \ldots \ldots \ldots$ & 3.24 & 1.87 & 1.08 & 0.62 & 1.08 & 0.62 \\
\hline$-22.50 \ldots \ldots \ldots \ldots \ldots$ & 3.24 & 1.87 & 1.08 & 0.62 & 1.08 & 0.62 \\
\hline$-22.25 \ldots \ldots \ldots \ldots \ldots$ & 5.41 & 2.42 & 1.80 & 0.81 & 1.80 & 0.81 \\
\hline$-22.00 \ldots \ldots \ldots \ldots \ldots$ & 14.06 & 3.90 & 4.69 & 1.30 & 4.69 & 1.30 \\
\hline$-21.75 \ldots \ldots \ldots \ldots \ldots$ & 12.98 & 3.75 & 4.33 & 1.25 & 4.33 & 1.25 \\
\hline$-21.50 \ldots \ldots \ldots \ldots$ & 21.63 & 4.84 & 7.21 & 1.61 & 7.21 & 1.61 \\
\hline$-21.25 \ldots \ldots \ldots \ldots \ldots$ & 24.87 & 5.19 & 8.29 & 1.73 & 8.29 & 1.73 \\
\hline$-21.00 \ldots \ldots \ldots \ldots \ldots$ & 21.63 & 4.84 & 7.21 & 1.61 & 7.21 & 1.61 \\
\hline \multicolumn{7}{|c|}{$\mathrm{DSS}+\mathrm{SSS}$} \\
\hline$-22.50 \ldots \ldots \ldots \ldots \ldots$ & 3.71 & 2.14 & $\ldots$ & $\ldots$ & 2.47 & 1.75 \\
\hline$-22.00 \ldots \ldots \ldots \ldots \ldots$ & 13.59 & 4.10 & 3.71 & 2.14 & 2.47 & 1.75 \\
\hline$-21.50 \ldots \ldots \ldots \ldots \ldots$ & 18.53 & 4.78 & 4.94 & 2.47 & 2.47 & 1.75 \\
\hline$-21.00 \ldots \ldots \ldots \ldots \ldots$ & 24.71 & 5.52 & 7.93 & 3.07 & 10.98 & 3.71 \\
\hline$-20.50 \ldots \ldots \ldots \ldots \ldots$ & 26.21 & 5.67 & 11.12 & 4.54 & 16.14 & 4.45 \\
\hline$-20.00 \ldots \ldots \ldots \ldots \ldots$ & 39.79 & 7.16 & 10.29 & 3.52 & 9.88 & 3.83 \\
\hline$-19.50 \ldots \ldots \ldots \ldots \ldots$ & 26.47 & 5.67 & 15.60 & 4.32 & 10.78 & 3.69 \\
\hline$-19.00 \ldots \ldots \ldots \ldots \ldots$ & 39.60 & 6.99 & 14.82 & 4.28 & 13.30 & 4.09 \\
\hline$-18.50 \ldots \ldots \ldots \ldots \ldots$ & 33.41 & 6.28 & 24.51 & 5.70 & 8.086 & 2.97 \\
\hline$-18.00 \ldots \ldots \ldots \ldots \ldots$ & 50.56 & 8.17 & 18.75 & 5.21 & 21.17 & 5.44 \\
\hline$-17.50 \ldots \ldots \ldots \ldots . . . .$. & 46.18 & 8.36 & 22.94 & 7.42 & 27.67 & 6.51 \\
\hline$-17.00 \ldots \ldots \ldots \ldots$ & 62.51 & 11.45 & $\ldots$ & $\ldots$ & 28.51 & 10.60 \\
\hline$-16.50 \ldots \ldots \ldots \ldots \ldots$ & 82.07 & 16.88 & $\ldots$ & $\ldots$ & 59.30 & 14.95 \\
\hline$-16.00 \ldots \ldots \ldots \ldots \ldots$ & 80.39 & 29.18 & $\ldots$ & $\ldots$ & 18.35 & 12.57 \\
\hline$-15.50 \ldots \ldots \ldots \ldots \ldots$ & $\ldots$ & $\ldots$ & $\ldots$ & $\ldots$ & 104.08 & 90.31 \\
\hline
\end{tabular}
circles). This leads to instability in parametric fits to LFs

TABLE 3

Observed LFs and Their Associated Errors for the Three Fields

Note.-LFs are in units of galaxies $\mathrm{mag}^{-1} \mathrm{Mpc}^{-2}$. Galaxies from both the extended SSS and DSS+SSS are used. A distance modulus of DM $=35.13\left(H_{0}=65 \mathrm{~km} \mathrm{~s}^{-1} \mathrm{Mpc}\right)$ is assumed for Coma. 

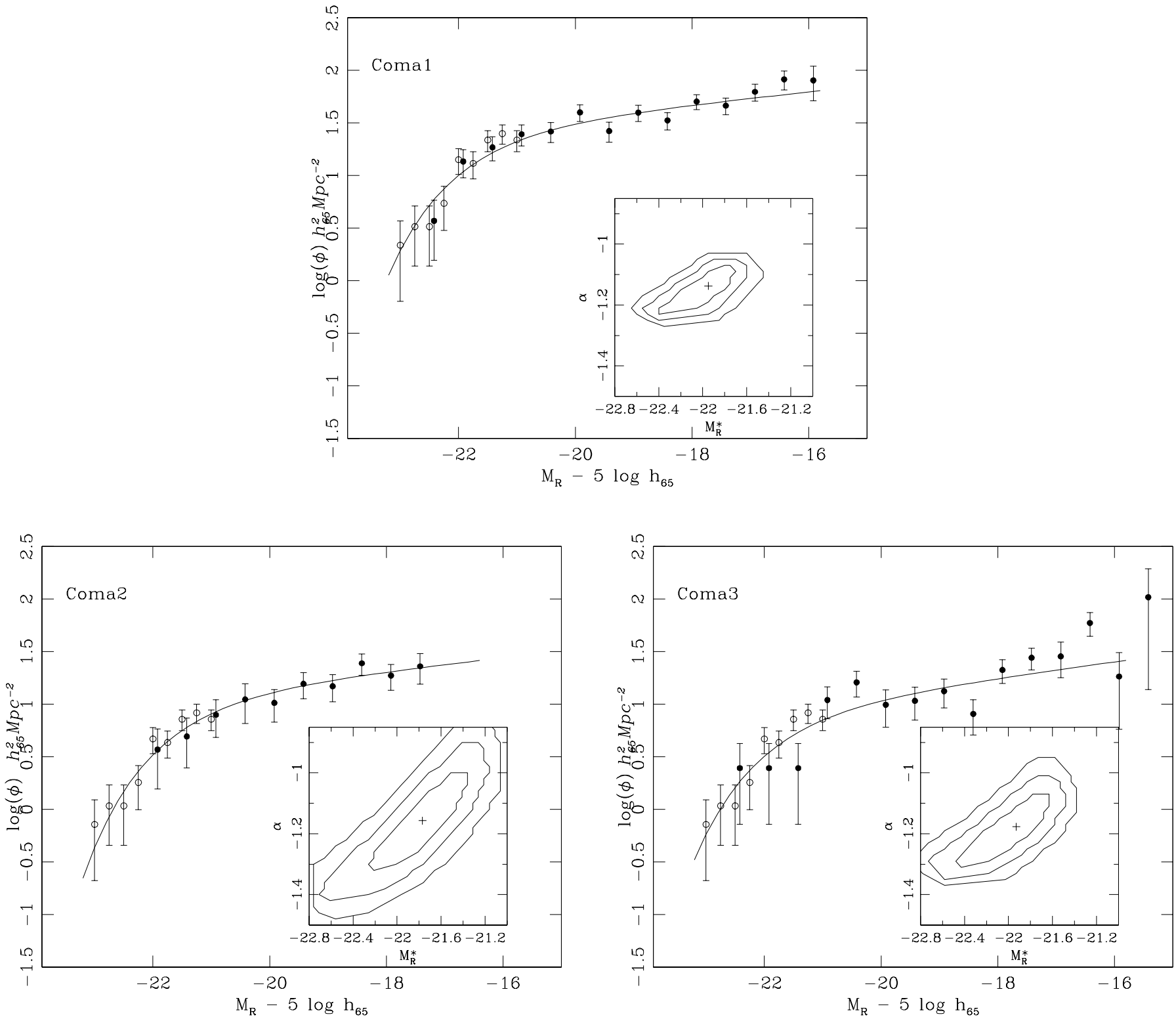

Fig. 5.-Observed LFs for the Coma 1, Coma 2, and Coma 3 fields. The DSS+SSS sample for a given field is shown by filled circles and the extended SSS sample (normalized to the DSS + SSS survey) by open circles. Schechter LF fits, as listed in Table 3, are also shown; 1,2 , and $3 \sigma$ error contours for $M_{R}^{*}$ and $\alpha$ are presented in the separate panel.

since the characteristic magnitudes and faint-end slopes are known to be correlated (see the next section for details). To overcome this, we construct the bright end of the LFs by combining the present survey (DSS+SSS) with an extension of the SSS sample, covering a larger area of the Coma Cluster. This extended survey covers an area of $1^{\circ}$ radius (corresponding to the field of view of $2 \mathrm{dF}$ ), centered on the Coma core and to the same depth as the SSS sample, substantially increasing the number of bright galaxies in the spectroscopic Coma survey. Here we assume that the shape of the bright end of the LFs is independent from their local environment, with the implication of this assumption discussed in $\S 4$ 4.1. However, photometry for galaxies in the extended SSS sample is only available in the $b$ and $r$ bands, measured from photographic plates (Godwin, Metcalfe, \& Peach 1983). To convert these to the photometric system used in this study, we use galaxies with available photom- etry in both systems and derive the empirical relation

$$
R=r+k_{1}(b-r)+k_{2},
$$

where $k_{1}=0.101 \pm 0.068$ and $k_{2}=0.143 \pm 0.037$ with $\mathrm{rms}=0.218 \mathrm{mag}$. This relation is subsequently used to convert magnitudes in the extended SSS sample to those of the MCCD photometric system of the present study.

The membership fraction is estimated for this sample, as discussed in the last section, and used to construct the LF over a $1^{\circ}$ radius region about the cluster center. The LF, binned in 0.25 mag intervals, is then normalized to those of the Coma 1, Coma 2, and Coma 3 fields, derived from the DSS and SSS samples, using galaxies with $M_{R}<-21 \mathrm{mag}$, and shown as open circles in Figure 5. This extends the bright end of the LFs to $M_{R}=-23$. By adopting the extended SSS sample here, we impose strong constraints on 
the bright end of the LF for each field while at the same time retaining the main advantage of the present sample in extending the Coma LF to the faintest possible magnitudes. The estimated bright end of the LF, derived from the extended sample and normalized to the LFs in each of the three fields, is also listed in Table 3. In the next section, parametric fits are carried out to the observed LFs over the entire magnitude range shown in Figure 5.

\subsection{Parametric Fits to Luminosity Functions}

We consider a Schechter parametric form for the observed LFs, expressed as

$$
\Phi_{s}(M)=\phi^{*} X^{\alpha+1} e^{-X},
$$

where $X=10^{-0.4\left(M-M^{*}\right)}$, with $M^{*}$ (characteristic magnitude), $\alpha$ (faint-end slope), and $\phi^{*}$ (normalization) the parameters to be determined (Schechter 1976).

Galaxies from the DSS and SSS, combined with those from the extended SSS sample, are used in the fit (both the filled and open circles in Fig. 5). As is clear from Table 3 and Figure 5, galaxies from the DSS+SSS and extended SSS samples overlap in the magnitude range $-22.50<$ $M_{R}<-21.00$. For galaxies over this interval, the average of $\phi(M)$ values is calculated in each magnitude bin and used to fit the LF. This allows the magnitude bins to be independent in the fitting process. Using only one of the DSS +SSS or extended SSS samples over their common magnitude interval will not affect the results. The fits are carried out over the luminosity range $-23<M_{R}-5 \log h_{65}<-16$ for Coma 1 and Coma 3 and $-23<M_{R}-5 \log h_{65}<-17.5$ for Coma 2. The fits for individual fields are shown in Figure 5 and presented in Table 4 . The reduced $\chi^{2}$ values, $\chi^{2} / \nu$, number of degrees of freedom, $\nu$, and the goodness-of-fit probability, $P\left(\chi^{2} \mid \nu\right)$, are also listed for each field. The 1, 2, and $3 \sigma$ error contours corresponding to each of the LFs are shown in Figure 5, with the $1 \sigma$ error estimates for LF parameters presented in Table 4 . The $\chi^{2}$ results in Table 4 indicate $P\left(\chi^{2} \mid \nu\right)>20 \%$ probability that the data, in all the three fields, are drawn from a Schechter LF, confirming that a Schechter form provides acceptable fits to individual LFs both at the core and in the outskirts of the cluster.

The procedure of using the bright end of the LF from the entire Coma Cluster to constrain that of the individual fields (located at different positions in Coma) makes the implicit assumption that the LFs at bright magnitudes are independent of their local environment. To explore the extent to which this assumption could affect overall shape of the LFs and their parametric form, we now fix $M^{*}$ values for each field to those measured and listed in Table 4 and carry out a two-parameter fit to DSS+SSS (i.e., the sample that extends to fainter magnitudes) to determine $\alpha$ and $\phi^{*}$. These results, together with their corresponding goodness of fit estimates, are also presented in Table 4. There is no difference (within the errors) in the faint-end slope and normalization of LFs between the two cases, with both providing acceptable fits to the observed LFs. The probability that these data are drawn from a parent Schechter LF is $P\left(\chi^{2} \mid \nu\right)>10 \%$, further confirming the previous result that a Schechter form provides acceptable fit to the LF at the core and outskirts of the Coma Cluster. This justifies the above assumption that the bright end of the LFs are similar among the three fields in the Coma.

Using the techniques developed and the results so far, we now concentrate on addressing the following three questions:

1. Is the LF the same at the core and outskirts of the Coma? How does it depend on luminosity?

2. Can the LF for the entire Coma Cluster be parameterized by a single form over its entire luminosity range?

3. Does the LF change, depending on the observed wavelength or color and surface brightness of galaxies?

\subsection{Environmental Dependence}

While the characteristic magnitudes for the LFs in the Coma 1, Coma 2, and Coma 3 fields are close (Table 4), there is evidence for a slight increase in the faint-end slope from -1.16 at the core (Coma 1) to -1.29 at the outskirts (Coma 3). However, this is only a $1 \sigma$ effect. It is also clear from the $\chi^{2}$ tests that a Schechter function form provides acceptable fits to the observed LFs in all the three fields and over the entire magnitude range covered. Moreover, this shows that all the three LFs have the same shape over their common luminosity range.

Given the similarity of LF shapes in different environments in the Coma Cluster, it is appropriate to sum the

TABLE 4

Results from the Parametric Fits to the Observed LFs in Coma 1, Coma 2 , Coma 3, And the Entire Coma Cluster

\begin{tabular}{|c|c|c|c|c|c|c|}
\hline Field & $M_{R}^{*}-5 \log h_{65}$ & $\alpha$ & $\begin{array}{c}\phi^{*} \\
\left(h_{65}^{2} \mathrm{Mpc}^{-2}\right)\end{array}$ & $\chi^{2} / \nu$ & $\nu$ & $\begin{array}{c}P\left(\chi^{2} \mid \nu\right) \\
(\%)\end{array}$ \\
\hline \multirow[t]{2}{*}{ Coma 1 ..................... } & \multirow{2}{*}{$-22.00_{-0.12}^{+0.13}$} & $-1.15_{-0.03}^{+0.03}$ & $27.3_{-1.5}^{+1.0}$ & 0.92 & 16 & 65 \\
\hline & & $-1.17_{-0.02}^{+0.03}$ & $25.4_{-10}^{+1.3}$ & 0.93 & 12 & 65 \\
\hline \multirow[t]{2}{*}{ Coma 2 ..................... } & \multirow{2}{*}{$-21.76_{-0.12}^{+0.15}$} & $-1.16_{-0.10}^{-0.02}$ & $11.9_{-0.9}^{+1.0}$ & 0.36 & 12 & 98 \\
\hline & & $\begin{array}{r}-0.10 \\
-1.20_{-0.04}^{+0.06}\end{array}$ & $10.5_{-10}^{+1.0}$ & 0.24 & 8 & 98 \\
\hline \multirow[t]{2}{*}{ Coma 3 .................... } & \multirow[t]{2}{*}{$-21.98_{-0.15}^{+0.15}$} & $-1.19_{-0.04}^{+0.04}$ & $8.9_{-1.7}^{+2.0}$ & 1.29 & 17 & 20 \\
\hline & & $\begin{array}{l}-1.29_{-0.03}^{-0.04} \\
-0.04\end{array}$ & $6.2_{-15}^{-1.7}$ & 1.63 & 13 & 10 \\
\hline Coma $3^{a} \ldots \ldots \ldots \ldots . . . . . . . .$. & $-21.92_{-0.15}^{+0.15}$ & $-1.31_{-0.05}^{+0.05}$ & $3.1_{-1.0}^{+1.2}$ & 1.72 & 17 & 5 \\
\hline All fields .................. & $\begin{array}{r}-0.15 \\
-21.79_{-0.09}^{+0.08}\end{array}$ & $-1.18_{-0.02}^{-0.05}$ & $9.5_{-0.6}^{-1.0}$ & 1.78 & 23 & 1 \\
\hline
\end{tabular}

Note.-Also listed are $1 \sigma$ error estimates. For each of the three fields, the LF parameters are estimated after constraining their bright end using data from the extended SSS sample (first line for each field). Then the $M_{R}^{*}$ values are fixed to these estimates and a two-parameter fit is performed to measure $\alpha$ and $\phi^{*}$ (second line for each field).

a Coma 3 LF with the NGC 4839 group removed. 
individual LFs to estimate the total LF, $\Phi(M)$, for the Coma Cluster as

$$
\Phi(M)=\Sigma_{i=1}^{3} \phi_{i}(M) .
$$

While this provides an accurate estimate of the faint end $\left(M_{R}>-18\right)$, it is only weakly constrained at the bright end $\left(M_{R}<-21\right)$, with large Poisson errors. Therefore, the total LF is derived here by combining galaxies in the DSS+SSS samples (Coma 1, Coma 2, and Coma 3), as measured by $\Phi(M)$, with those in the extended SSS (all galaxies in the shallow spectroscopic survey covering an area of $1^{\circ}$ diameter in the Coma). The membership fraction is measured as discussed in the last section. The two LFs are normalized over the magnitude range $-22.5<M_{R}<-21$, with only the extended SSS sample at $M_{R}<-17.75$ used for fitting to the parametric form. The total LF is then derived over a magnitude range $-23<M_{R}-5 \log h_{65}<-16$ and presented in Figure 6 . The total $\mathrm{LF}$ over a $1^{\circ}$ radius region about the cluster center is listed in Table 5, with its corresponding parametric fit presented in Table 4.

The reduced $\chi^{2}$ value, corresponding to the LF from all fields (last line in Table 4), indicates a small probability $\left[P\left(\chi^{2} \mid \nu\right) \sim 1 \%\right]$ that the data are drawn from a Schechter LF. We now consider the possibilities that might contribute to this. The total LF in Figure 6 is constructed assuming the LF for galaxies covering the entire $1^{\circ}$ diameter in the Coma to have the same shape as those in the three (Coma 1, Coma 2, and Coma 3) fields. However, it is likely that this is not the case for other regions of the Coma Cluster (because of differences in local densities), affecting the bright end of the LF. Indeed, constraining the sample to only the three fields studied here (DSS+SSS galaxies located in the Coma 1, Coma 2, and Coma 3 fields) increases the probability to $P\left(\chi^{2} \mid \nu\right) \sim 10 \%$, with no significant change in the estimated LF parameters listed in Table 4. Moreover, differences in normalizations $\left(\phi^{*}\right.$-values) between the three fields are likely to result in a poor fit to a Schechter LF form once the

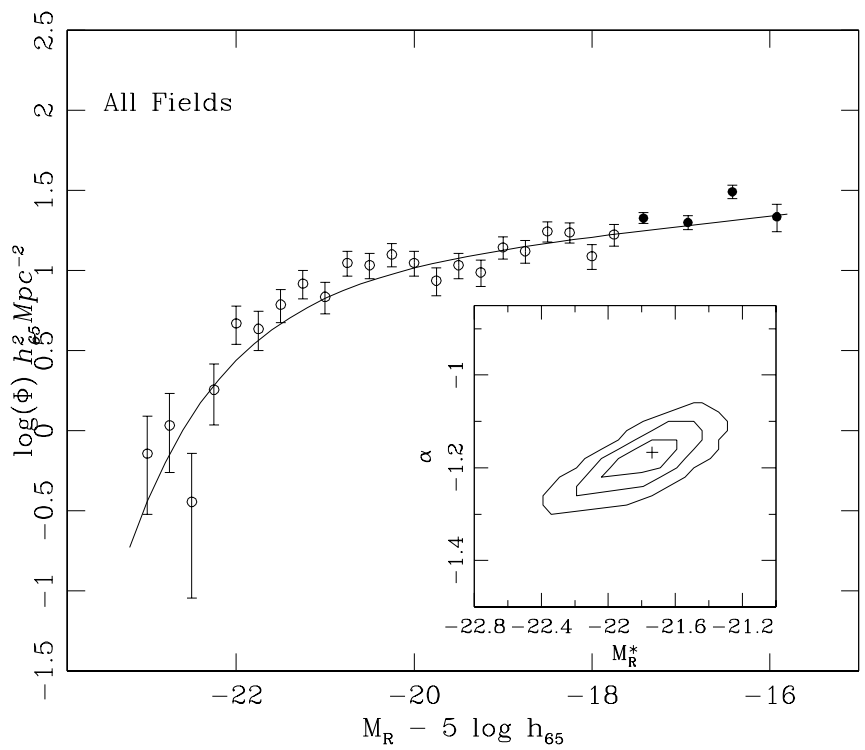

Fig. 6.-Total $R$-band LF, covering an area of $1^{\circ}$ radius of the Coma Cluster. A combined sample from the SSS (open circles) and DSS ( filled circles) is used. The Schechter LF fit is also presented. $M_{R}^{*}$ and $\alpha$ error contours at 1,2 , and $3 \sigma$ levels are also shown.
TABLE 5

Total LF $[\Phi(M)]$ For the Entire Coma Cluster

\begin{tabular}{|c|c|c|c|c|c|}
\hline$M_{R}$ & $\Phi^{R}(M)$ & $\Delta\left[\Phi^{R}(M)\right]$ & $M_{B}$ & $\Phi^{\mathrm{B}}(M)$ & $\Delta\left[\Phi^{B}(M)\right]$ \\
\hline \multicolumn{6}{|c|}{ Extended SSS } \\
\hline$-23.00 \ldots \ldots$ & 0.72 & 0.51 & -21.75 & 0.72 & 0.51 \\
\hline$-22.75 \ldots \ldots$ & 1.08 & 0.62 & -21.25 & 1.44 & 0.72 \\
\hline$-22.50 \ldots \ldots$ & 0.36 & 0.36 & -21.00 & 0.72 & 0.51 \\
\hline$-22.25 \ldots \ldots$ & 1.80 & 0.81 & -20.75 & 2.16 & 0.88 \\
\hline$-22.00 \ldots \ldots$ & 4.69 & 1.30 & -20.50 & 2.52 & 0.95 \\
\hline$-21.75 \ldots \ldots$ & 4.33 & 1.25 & -20.25 & 3.96 & 1.19 \\
\hline$-21.50 \ldots \ldots$ & 6.13 & 1.49 & -20.00 & 5.77 & 1.44 \\
\hline$-21.25 \ldots \ldots$ & 8.29 & 1.73 & -19.75 & 9.01 & 1.80 \\
\hline$-21.00 \ldots \ldots$ & 6.85 & 1.57 & -19.50 & 7.57 & 1.65 \\
\hline$-20.75 \ldots \ldots$ & 11.17 & 2.01 & -19.25 & 10.81 & 1.97 \\
\hline$-20.50 \ldots \ldots$ & 10.81 & 1.97 & -19.00 & 12.61 & 2.13 \\
\hline$-20.25 \ldots \ldots$ & 12.61 & 2.13 & -18.75 & 15.14 & 2.33 \\
\hline$-20.00 \ldots \ldots$ & 11.17 & 2.01 & -18.50 & 11.17 & 2.01 \\
\hline$-19.75 \ldots \ldots$ & 8.65 & 1.77 & -18.25 & 10.09 & 1.91 \\
\hline$-19.50 \ldots \ldots$ & 10.81 & 1.97 & -18.00 & 9.37 & 1.84 \\
\hline$-19.25 \ldots \ldots$ & 9.73 & 1.87 & -17.75 & 11.89 & 2.07 \\
\hline$-19.00 \ldots \ldots$ & 13.95 & 2.25 & -17.50 & 17.21 & 2.51 \\
\hline$-18.75 \ldots \ldots$ & 13.21 & 2.19 & -17.25 & 15.19 & 2.36 \\
\hline$-18.50 \ldots \ldots$ & 17.57 & 2.54 & -17.00 & 14.84 & 2.35 \\
\hline$-18.25 \ldots \ldots$ & 17.29 & 2.52 & -16.75 & 13.88 & 2.28 \\
\hline$-18.00 \ldots \ldots$ & 12.30 & 2.19 & -16.50 & 16.25 & 2.51 \\
\hline$-17.75 \ldots \ldots$ & 16.80 & 2.68 & -16.25 & 12.28 & 2.26 \\
\hline \multicolumn{6}{|c|}{ DSS Survey } \\
\hline$-17.50 \ldots \ldots$ & 21.23 & 1.75 & -16.00 & 12.28 & 1.14 \\
\hline$-17.00 \ldots \ldots$ & 19.96 & 2.11 & -15.50 & 13.31 & 1.43 \\
\hline$-16.50 \ldots \ldots$ & 31.00 & 3.06 & -15.00 & 18.93 & 1.97 \\
\hline$-16.00 \ldots \ldots$ & 21.65 & 4.30 & -14.50 & 11.62 & 2.13 \\
\hline
\end{tabular}

Note.-Galaxies from the SSS sample $\left(M_{R}<-17.5, M_{B}<-16.00\right)$ are combined with data from DSS $\left(M_{R}>-17.5, M_{B}>-16.00\right)$. Errors are estimated as discussed in the text.

fields are combined. Alternatively, it is possible that a twocomponent LF (i.e., Gaussian+Schechter) is needed to model the total LF for the Coma Cluster (Biviano et al. 1995; Yagi et al. 2002).

The observed faint-end slope of $-1.18_{-0.02}^{+0.04}$ found here, while consistent with individual fields (Coma 1, Coma 2, and Coma 3), is relatively shallower than that obtained from other studies of the Coma Cluster LF (Trentham 1998; Secker \& Harris 1996; Beijersbergen et al. 2002). However, the spectroscopic LF here has a much brighter magnitude limit $\left(M_{R}=-16\right)$. Moreover, the measurements based on photometric surveys are mostly unconstrained at faint magnitudes because of small-number statistics, incompleteness, uncertain background correction, and contamination by globular clusters (see $\S 5$ for details).

Study of the dependence of LFs on their local environments here could be hampered by the presence of the NGC 4839 group in the Coma 3 field. This is examined by removing galaxies associated with this group from the Coma 3 sample and then measuring its LF. Defining the NGC 4839 group as galaxies within a circle of radius $9^{\prime}$ centered on the cD galaxy NGC 4839 (Beijersbergen et al. 2002), we construct the Coma $3 \mathrm{LF}$ after removing these objects and then carry-out a parametric fit to the Schechter LF form. The result is also listed in Table 4 and shows a slight increase $(1 \sigma)$ in the faint-end slope of the Coma LF from the core to outskirts. Spectroscopic data on another field in the outskirt 
of the Coma Cluster at a similar distance from the center as Coma 3 are needed to accurately measure changes in the faint-end slope with radius.

\subsection{Wavelength Dependence}

The $B$-band LF for the entire Coma Cluster is constructed and presented in Figure 7, following the same procedure discussed in $\S 4$. The extended SSS (covering $1 \mathrm{deg}^{2}$ area of the Coma) and DSS samples are used with the completeness functions estimated in the same way as for the $R$-band LF. The $B$-band magnitudes that were not available for a fraction of galaxies in the extended SSS survey are estimated using their $R$-band magnitudes (measured from photographic plate $b$ - and $r$-band data in $\S 4$ ) and $B-R$ colors. The total $B$-band LF is also tabulated in Table 5.

The spectroscopic sample in this study was selected in the $R$ band. This was motivated because the light at longer wavelengths is dominated by the evolved stellar population and because the Coma Cluster is rich in early-type evolved galaxies. Therefore, we expect a bias against the very blue and faint galaxies, leading to a flatter faint-end slope for the $B$-band LF here. Having this in mind, the $B$-band LF is fitted to a Schechter form, with the best fit and 1, 2, and $3 \sigma$ error contours presented in Figure 7. We estimate $\quad M_{B}^{*}=-19.95_{-0.10}^{+0.15}+5 \log h_{65}, \quad \alpha=-0.96_{-0.02}^{+0.01}$, $\phi^{*}=16.3_{-0.1}^{+0.1} h_{65}^{2} \mathrm{Mpc}^{-2}$, and $\chi^{2} / \nu=1.16$. This corresponds to a $P\left(\chi^{2} \mid \nu\right)=25 \%$ probability that a Schechter LF form is a good representation of the data.

The total $B$-band LF in Figure 7 shows a dip at $M_{B}=-18$ mag. A similar gap with smaller amplitude has been observed in the total $R$-band LF (Fig. 6) at almost the same magnitude, $M_{R}^{*}=-19.5$, shifted by the mean color of galaxies in this sample $(\langle B-R\rangle=1.5)$. Although this is only a $1 \sigma$ effect and likely caused by Poisson statistics, a detailed study of this feature is useful since it gives clues toward the overall shape of the LF. Such a behavior has also been confirmed from other studies at almost the same magnitude in the rest-frame $B$-band LFs of both nearby (Biviano et al. 1995) and intermediate-redshift (Dahlen et al. 2003) clusters. Studying the $R$-band LFs for a photometric sample of

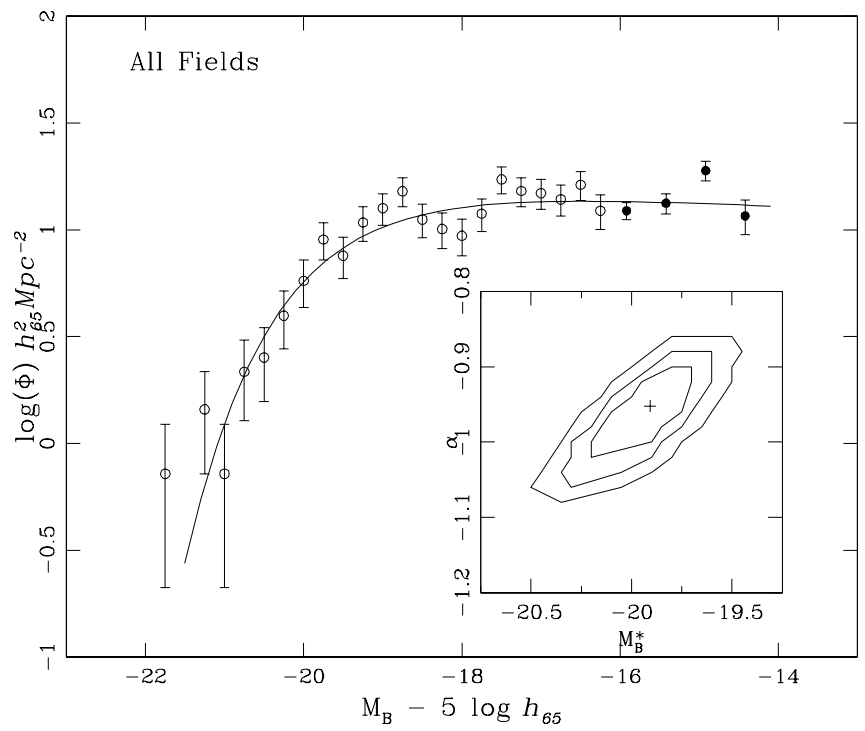

FIG. 7.-Same as Fig. 6, but for the $B$-band LF
10 clusters at different redshifts and with different richness classes, Yagi et al. (2002) show evidence for a dip in the cluster LFs at the same magnitude as here. After dividing their sample into type specific groups, consisting of elliptical $\left(r^{1 /}\right.$ 4-like profile) and spiral (exponential-like profile), they conclude that the observed dip is almost entirely due to contribution from the early-type $\left(r^{1 / 4}-\right.$ like $)$ galaxies to the total LF. They also find that clusters with larger velocity dispersions have more distinct dips, perhaps indicating that the amplitude of the dip depends on the dynamical state of the cluster and, consequently, on the dominant population of galaxies in that cluster.

To explore the effect of this gap on the general shape of the LF, we now remove galaxies in the range $-18.5<M_{B}<-17.75$, which contribute to the gap in Figure 7, and then fit the LF to a Schechter form. We find $M_{B}^{*}=-19.74 \pm 0.10+5 \log h_{65}, \quad \alpha=-0.90 \pm 0.02, \phi^{*}=$ $20.7 \pm 0.1 h^{2} \mathrm{Mpc}^{-2}$, and $\chi^{2} / \nu=0.97$. This increases the probability of a Schechter LF form being a good representation of the data to $P\left(\chi^{2} ; \nu\right)=50 \%$. Given this result, it is likely that a two-component shape for the LF, consisting of a Gaussian, representing giant galaxies $\left(M_{B}<-18\right)$, and a power law, representing the dwarf population $\left(M_{B}>-18\right)$, may well be appropriate for the composite LF over its entire magnitude range.

\subsection{Color Dependence}

The survey performed here is extensive enough to allow a study of the LFs in both different environments in the Coma Cluster and color intervals. This requires knowledge of the membership fraction in $B-R$ color intervals. To minimize statistical uncertainties, we use galaxies from all the fields (in both the DSS and SSS samples) to derive a common selection function, assuming no spatially dependent selection bias.

Two color intervals are adopted, corresponding to blue $(B-R<1.35)$ and red $(B-R>1.35)$ galaxies. This color is chosen so that it is consistent with the average color of an intermediate-type spiral (i.e., Sc), allowing for clear separation between star-forming (blue) and evolved (red) populations. Moreover, this provides a sufficiently large number of blue and red galaxies for statistical analysis. The blue galaxies here are also found to have strong emission lines, further confirming that they are undergoing star formation activity. The membership fractions, derived for the two color bins following the procedure explained in $\S 3$, are presented in Figure 8. It is clear that, for both blue and red galaxies, the survey is $80 \%$ complete to $R \sim 16$, dropping to $40 \%$ completeness at $R \sim 18.5$. Using membership fractions, the LFs are derived in $B-R<1.35$ (star-forming) and $B-R>1.35$ (evolved) intervals and presented in Figure 9 for the three fields separately and all the fields together.

The evolved galaxies in Coma 1 have a surface density that is an order of magnitude higher than that of starforming galaxies, with the difference decreasing toward the outskirts (the Coma 2 and Coma 3 fields). The sudden increase in the slope of the Coma 3 field LF for red galaxies at $M_{R}>-18$ is due to the contribution from the NGC 4839 group. The $B-R$ color distribution for spectroscopically confirmed members of this group (Fig. 10) shows a significant number of these galaxies having $B-R>1.35$. The similarity between the slope of the LFs for star-forming 


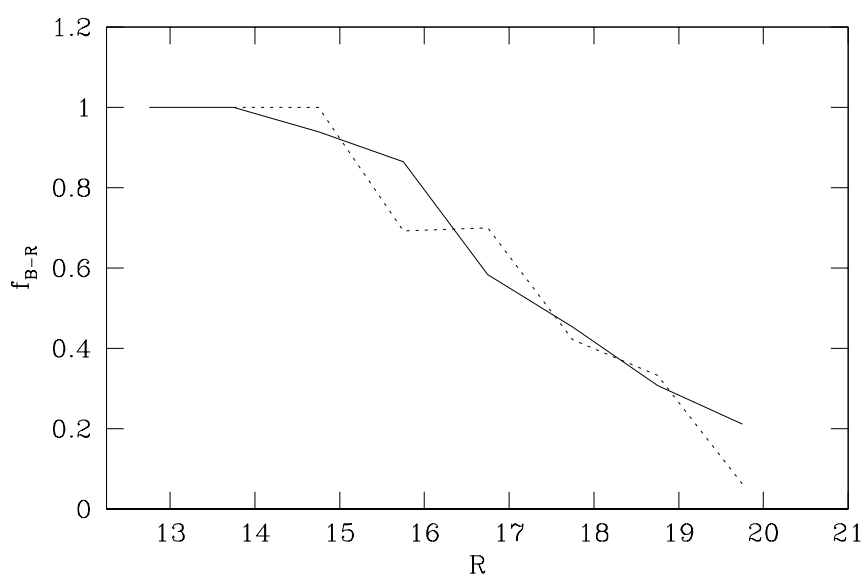

FIG. 8.-Membership fractions in $B-R$ color intervals for galaxies in $\mathrm{DSS}+\mathrm{SSS}$ samples; $B-R>1.35$ (solid line) and $B-R<1.35$ (dashed line).

galaxies in different environments contradicts the result from Beijersbergen et al. (2002), who found a relatively steeper $U$-band LF in the outer part of the Coma Cluster. The difference here is likely due to selection of the present sample in the red passband, statistical fluctuations in both studies, and uncertainties in background subtraction in the Beijersbergen et al. sample.

Recently, it was shown that for a given spectral type of galaxies, the faint-end slope of the cluster LF is steeper than that in the field (De Propris et al. 2002). This is in apparent contradiction to the result here, in which similar LFs are found for both the blue and red populations in the core (Coma 1) and outskirts (Coma 3) of the Coma. However, clusters used in De Propris et al. (2002) cover a wide range in richness, with many being as rich as the Coma 3 field here. This indicates a larger density contrast between the cluster sample of De Propris et al. and the general field, compared

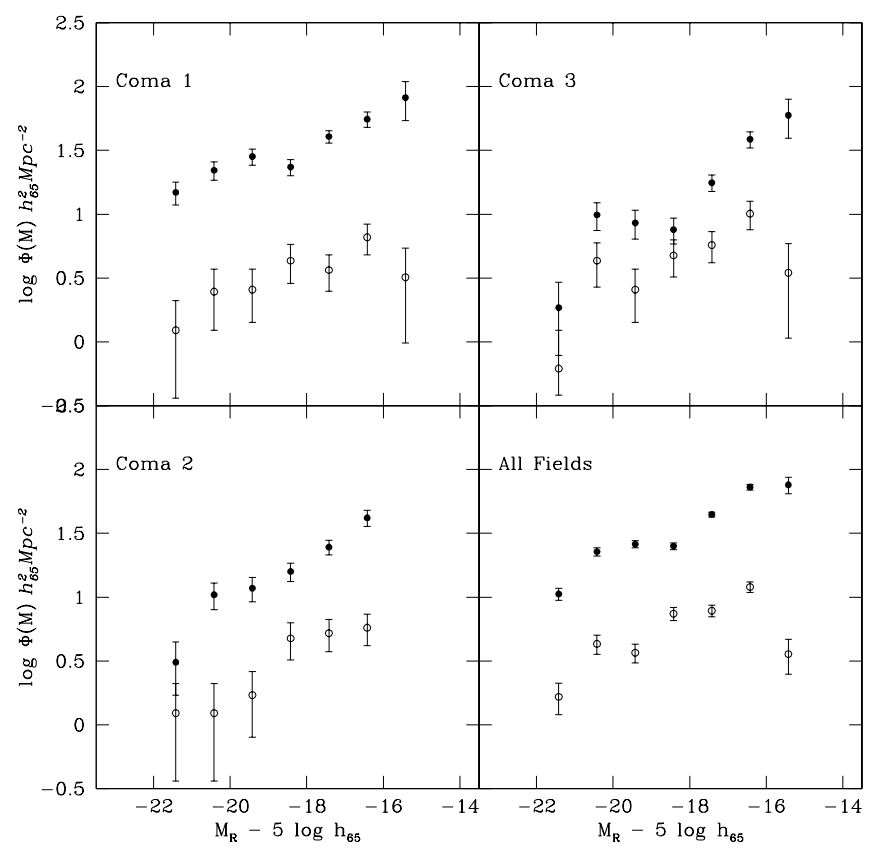

FIG. 9.-LFs in $B-R$ color intervals for galaxies at the core and outskirts of the Coma Cluster. $B-R>1.35$ (filled circles) and $B-R<1.35$ (open circles). The error bars are estimated as discussed in the text.

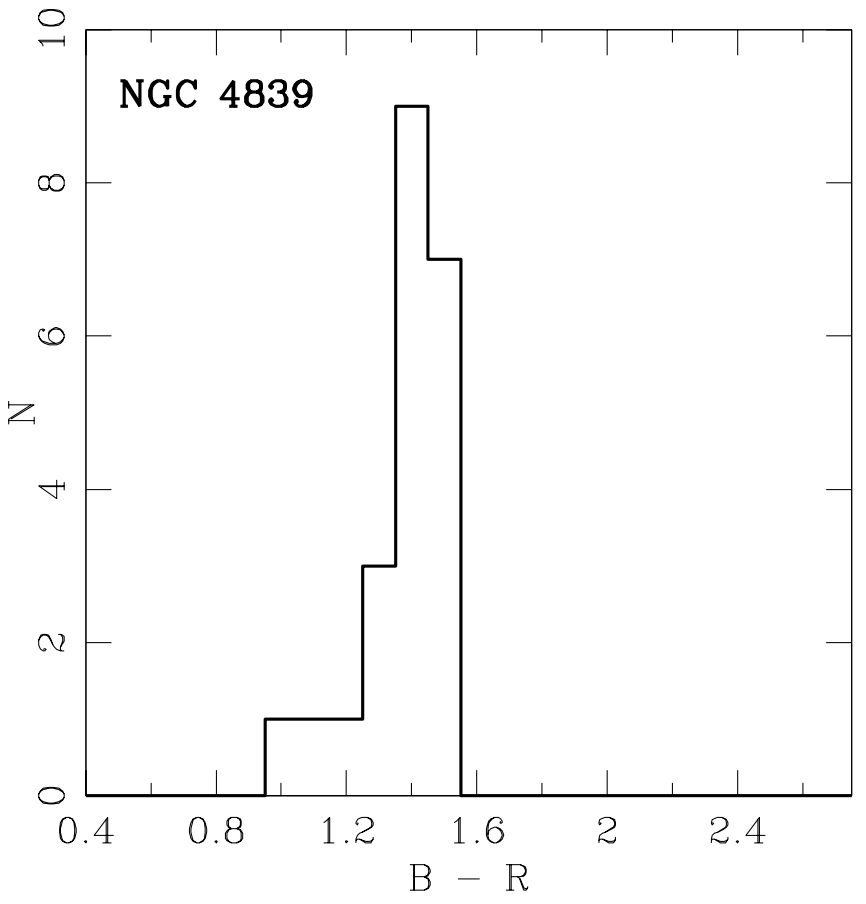

FIG. 10. $-B-R$ color distribution for spectroscopically confirmed members of the NGC 4839 group in the Coma 3 field.

to the Coma 1 and Coma 3 fields. Moreover, the results from the two studies are not directly comparable since there is only a loose relation between colors and spectral types used in these two studies.

\subsection{Surface Brightness Dependence}

The surface brightness distributions in Figure 3 show that, on average, the cluster members have a relatively brighter surface brightness than field galaxies. This implies that higher surface brightness galaxies tend to reside in environments with relatively larger densities. Figure 11 presents luminosity distributions for spectroscopically confirmed members of the Coma Cluster in three surface brightness intervals: $\mu_{\text {eff }}<20 \mathrm{mag} \operatorname{arcsec}^{-2}, 20 \mathrm{mag} \operatorname{arcsec}^{-2}<$ $\mu_{\text {eff }}<22 \mathrm{mag} \mathrm{arcsec}^{-2}$, and $\mu_{\text {eff }}>22 \mathrm{mag} \mathrm{arcsec}^{-2}$. There is a clear trend in the sense that low surface brightness galaxies have fainter magnitudes, reflecting a monotonic magnitude-surface brightness relation. Such a relation has also been found for field galaxies (Brown et al. 2001; Cross et al. 2001). However, there is also a considerable number of low surface brightness $\left(\mu_{\text {eff }}>22\right)$ galaxies in all the Coma fields $(>50 \%$; Fig. 11). This indicates a low surface brightness population that dominates the faint end of the LF, in agreement with results from Andreon \& Cuillandre (2002) and Sprayberry et al. (1997).

\section{COMPARISON WITH OTHER STUDIES}

Recent studies of the Coma Cluster LF have led to the discovery of steep, faint-end slopes in both optical (Thompson \& Gregory 1993; Secker \& Harris 1996; Trentham 1998) and near-IR (Mobasher \& Trentham 1998; De Propris et al. 1998; Rines et al. 2001) wavelengths. All these studies are based on photometric surveys with statistical background corrections from control fields. Recently, 


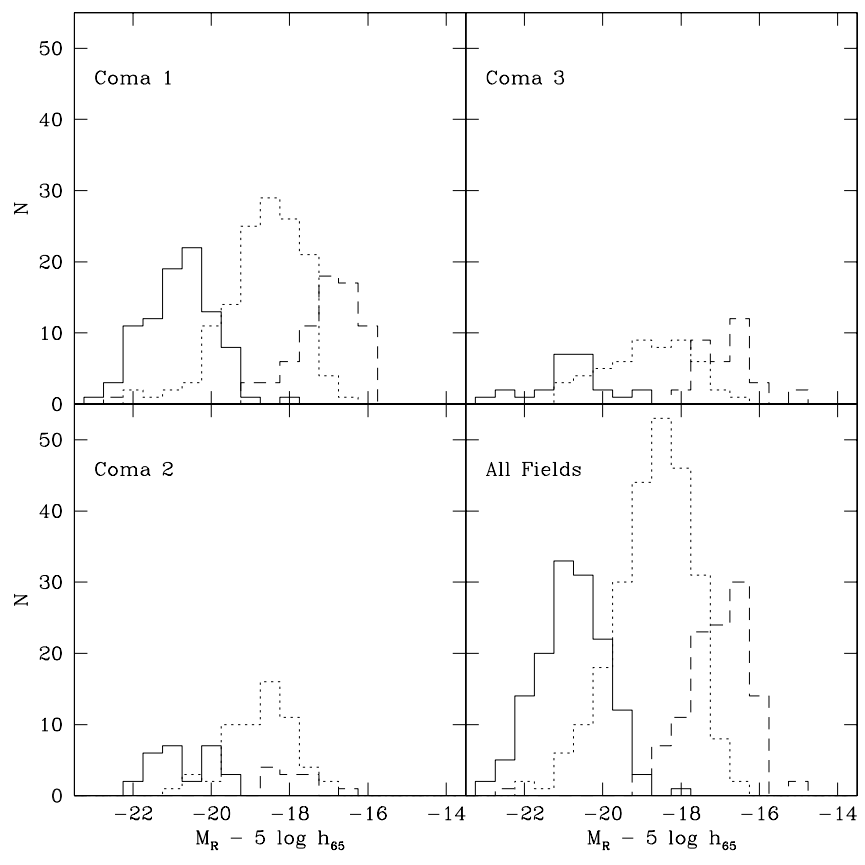

FIG. 11.-Absolute magnitude distributions in effective surface brightness intervals for galaxies at the core, outskirts, and the entire Coma Cluster; $\mu_{\mathrm{eff}}<20 \mathrm{mag} \operatorname{arcsec}^{-2}$ (solid line), $20 \mathrm{mag} \operatorname{arcsec}^{-2}<\mu_{\mathrm{eff}}<22$ mag $\operatorname{arcsec}^{-2}$ (dotted line), and $\mu_{\mathrm{eff}}>22 \mathrm{mag} \mathrm{arcsec}^{-2}$ (dashed line).

this technique has gained popularity with the advent of large-format and highly sensitive panoramic detectors, allowing a more statistically representative measure of background contamination.

Using photographic plates, Thompson \& Gregory (1993) determined the LF to $B \sim 20\left(M_{B} \sim-15\right)$. They found a steepening of the slope at faint magnitudes $(\alpha=-1.4)$. This was followed by Bernstein et al. (1995), who used CCD surveys, extending the Coma LF to much fainter limits $\left(M_{R} \sim-9.5\right)$ over a smaller region. These authors also found a steep faint-end slope $(\alpha=-1.8)$. However, their result was significantly affected by globular cluster contamination in the halo of NGC 4874, making their unconstrained LF fainter than $M_{R}=-14$. A deeper survey over a much larger area (700 $\left.\mathrm{arcmin}^{2}\right)$ by Secker \& Harris (1996) also found an LF that rises to $M_{R}=-14$ and flattens thereafter. The flat bright-end, followed by steep faint-end slope, was further confirmed by Lobo et al. (1997).

Recently, Beijersbergen et al. (2002) carried out a multiwavelength $(\mathrm{UBr})$ study of LF in the Coma Cluster. They found $M_{R}=-21.47_{-0.17}^{+0.12}+5 \log h_{65}$ and $\alpha=-1.16$, with a steeper faint-end slope at larger radii from the cluster center. The increase in the faint-end slope of the $U$-band LF was interpreted as being due to a population of star-forming dwarf galaxies. No such increase in the number density of this population is found, as is clear from the $B$-band LF in Figure 7. Also, Andreon \& Cuillandre (2002) carried out a wide-area $\left(650 \mathrm{arcmin}^{2}\right)$ photometric survey of the Coma Cluster. Combining this survey with Hubble Space Telescope observations, they confirmed that unresolved globular clusters could be mistakenly classified as dwarf galaxies at faint magnitudes, causing the observed steepness of the LF faint-end slope. Correcting for this, they derived $\alpha=-1.4$, with both the shape and slope of the LF depending on color. They also derived LFs in surface brightness intervals and found low surface brightness galaxies as the main contributor at fainter magnitudes, as expected from, for example studies of the Local Group (Mateo 1998). This result is in close agreement with that found in $\S 4.5$, using a spectroscopic sample, and that in Trentham (1998) from a photometric survey.

Trentham (1998) avoided some of the problems affecting the above studies by using a deeper control field to improve background estimates and contamination (at the faint end) by globular clusters. Surveying a large area $\left(674 \mathrm{arcmin}^{2}\right)$ to deep $(R=24)$ limits, he found a steep faint-end slope $(\alpha=-1.7)$ at $M_{R}=17.6$ followed by a turnover in the LF at $M_{R}=15.6$, in both the core $(r<200 \mathrm{kpc})$ and the outskirt $(r>200 \mathrm{kpc})$ fields. The population of galaxies at $R \sim 19$ was found to be dwarf spheroidals with $B-R=1.3$. The narrow spread in $B-R$ colors here was taken as evidence for a homogeneous population of dwarfs in the Coma, in agreement with recent results. However, the slope and general shape of the LF at faint magnitudes only depend on a few points with large error bars, making this part of the LF essentially unconstrained.

All the above studies are based on photometric surveys with statistical background corrections. The faint-end slopes of the LFs from photometric surveys discussed above are relatively steeper than that found from the present study, based on a spectroscopic survey. This is mostly due to the depth of photometric surveys, extending to $M_{R} \sim-12$, compared to the shallower spectroscopic survey here $\left(M_{R}<-16\right)$, with the upturn in the faint-end slope appearing at $M_{R}>-16$. Moreover, it is possible that a combination of uncertain background correction and contamination by globular clusters, especially at faint magnitudes, may have led to the steep faint-end slopes found in studies based on photometric surveys. However, in a recent study, using deep simulated catalogs of cluster galaxies constructed from a flat LF $(\alpha=-1)$, Valotto, Moore, \& Lambas (2001) show a strong tendency toward a steep faint-end slope for LFs $(\alpha>-1.5)$ in clusters selected in two dimensions. This indicates that the cause of the observed steep faint-end slope in clusters, seen in photometric surveys, is due to the projection effect.

Using a sample of 205 spectroscopically confirmed galaxies with $b_{26.5}<18$ at the $48 \times 25 \operatorname{arcmin}^{2}$ center of the Coma Cluster, Biviano et al. (1995) found that a single Schechter function cannot adequately fit the LF of the Coma Cluster. They found that a combination of Schechter and Gaussian forms provides a significantly better fit to the LF. However, the Biviano et al. data do not strongly constrain the faint end of the LF, with their spectroscopic sample becoming progressively incomplete toward fainter magnitudes.

It is useful to compare the Coma LF derived here with those in other rich clusters (Virgo), less evolved clusters (Ursa Major), and the general field. A recent study of the Virgo LF finds a steep faint-end slope of $\alpha=-1.6$, based on photometric $B$-band CCD survey (Trentham \& Hodgkin 2002). Although steep, this is significantly shallower than the slope of $\alpha=-2.2$, found in the $R$ band by Phillipps et al. (1998). Despite the fact that these are based on power-law fits overrestricted magnitude range, both these studies show the LF becoming relatively flat after the rise at the bright end and before steepening toward fainter magnitudes. However, because of the dominance of star over galaxy counts and steep galaxy counts in the Virgo Cluster, measurement for 
the LF of this cluster is uncertain. Therefore, the difference in the faint-end slopes found between these studies shows the uncertainty involved in measuring background contamination when using photometric surveys in clusters (the optimal redshift for background subtraction is found to be $z \sim 0.15$ [Driver, Windhorst, \& Griffiths 1995]), different selection effects, and methodologies. It is also worth noting that because of the prolate shape of the Virgo Cluster (Yasuda, Fukugita, \& Okamura 1997; West \& Blakeslee 2000; Arnaboldi et al. 2002), an accurate determination of its LF is further complicated. The Ursa Major LF is found to be significantly different from those in richer clusters like Virgo and Coma (Trentham, Tully, \& Verheijen 2001). For example, it does not show the steep increase at the faint end, indicating a relatively smaller population of dwarfs with respect to giants.

Recently, there have been two comprehensive studies of the field LF from the Sloan Digital Sky Survey (SDSS; Blanton et al. 2001) and 2df Galaxy Redshift Survey (2dFGRS; Cross et al. 2001; Norberg et al. 2002). Because of the need for spectroscopy in estimating the LF for field galaxies, these normally span a brighter magnitude range than their cluster counterparts, derived from photometric surveys with statistical background correction. This, combined with the fact that these two studies are based on different filters and magnitude scales compared to present work, makes a detailed and quantitative comparison difficult. Nevertheless, the faint-end slope of the Coma LF from this study $\left(-1.18_{-0.02}^{+0.04}\right)$ agrees with those from the SDSS $(-1.24 \pm 0.05)$ and $2 \mathrm{dFGRS}(-1.21 \pm 0.03)$ field LFs. Moreover, the SDSS reveals a turnover in the faint end of the LF for red galaxies and a relatively steep LF for blue galaxies. This effect is not observed in Figure 9. However, in both the SDSS and 2dFGRS, strong correlations are found between luminosity and surface brightness, in qualitative agreement with results from the present study.

\section{DISCUSSION}

The shape of the LF at faint magnitudes depends on the efficiency with which gas is converted into stars in low-mass systems (Dekel \& Silk 1986; Efstathiou 2000). For example, dark halos in dense environments could collect gas (through intracluster pressure) and be turned into stars, but dark halos in unevolved (diffuse) environments could not, leading to more low-luminosity galaxies (dwarfs) per unit total mass in evolved environments compared to the unevolved or field environment (Tully et al. 2002; Somerville 2002). However, this effect is contrasted with the ongoing process of galaxy interaction and tidal stripping in dense regions of clusters, resulting in a decrease in the number of faint galaxies and, hence, a flatter faint-end slope for the LF, while the reverse is the case in areas with small galaxy densities (poor clusters and groups; López-Cruz et al. 1997; Phillipps et al. 1998; Adami et al. 2000; Conselice 2002). The observed faint-end slope of the LF is likely to be fixed by a competition between these two effects.

The $R$-band LFs measured here show a slight increase in their faint-end slope from $-1.16_{-0.02}^{+0.03}$ in Coma 1 (inner region) to $-1.21_{-0.04}^{+0.06}$ in Coma 2 (intermediate region) and $-1.29_{-0.03}^{+0.04}$ in Coma 3 (outer region). However, this is only a $1 \sigma$ effect. Contribution from the NGC 4839 group to the faint-end slope of the Coma 3 field is examined by removing galaxies associated with this group and estimating the Coma
3 LF again. This does not significantly change the faint-end slope of the Coma 3 field. Recently, using a spectroscopic sample of 60 clusters with different richness classes, selected from the 2dFGRS, De Propris et al. (2002) found a composite LF with $\alpha=-1.25 \pm 0.03$, similar to that of the field LF. However, for a given spectral type of galaxies, they found the cluster LF to be significantly steeper than the LF for field galaxies of the same spectral type. The $R$-band luminosity of galaxies, used in the present study, is sensitive to the old stellar population (i.e., mass function) and, hence, not significantly affected by ongoing star formation. Therefore, the similarity of the LFs between the core and outskirts of the cluster, and their closeness to the field LFs, estimated from SDSS (Blanthon et al. 2001) and 2dFGRS (Cross et al. 2001; Norberg et al. 2002), suggests that the average luminosity produced for a given galaxy mass is little affected by the processes that make significant changes to the morphologies and spectral types of individual galaxies. This is in qualitative agreement with results in De Propris et al. (2002).

The similarity of the faint-end slopes of the $R$-band LF between core and outskirts of the Coma Cluster is in agreement with the results in Trentham (1998), who uses a significantly deeper photometric survey, extending to $M_{R}=-12$. However, Beijersbergen et al. (2002) found an increase in the faint-end slope of the $U$-band LF with radius from the center of the Coma Cluster, indicating an abundance of star-forming galaxies in low-density regions. Considering the observed LFs from these studies, the theoretical prediction of a turnover in the LF at faint magnitudes due to the suppression of star formation in low-mass galaxies (Efstathiou 1992; Chiba \& Nath 1994; Thoul \& Weinberg 1995), is not supported. However, because of the limited depth of the spectroscopic survey in this paper $\left(M_{R}<-16\right)$ and uncertainties in background correction and normalization in photometric surveys (Trentham 1998; Beijersbergen et al. 2002), one cannot constrain these models. A singlecomponent Schechter function form gives acceptable fits to the observed LFs over a range of 7 mag $\left(-23<M_{R}-\right.$ $\left.5 \log h_{65}<-16\right)$ at both the core and outskirts of the Coma Cluster. However, in a recent study, Yagi et al. (2002) showed that the composite LF from 10 nearby clusters is well described by a Schechter form, while the LFs for individual clusters are not. Furthermore, they found the composite LF to have a steeper faint-end slope compared to the general field, in contrast to the present study and the results from De Propris et al. (2002). However, this is less than a $2 \sigma$ effect and not statistically significant.

The characteristic magnitudes of LFs derived here agree with recent estimates from Beijersbergen et al. $\left(2002 ; \quad M_{R}^{*}=-21.91_{-0.34}^{+0.26}+5 \log h_{65} ; \quad M_{B}^{*}=-20.03_{-0.40}^{+0.36}\right.$ $\left.+5 \log h_{65}\right)$, after conversion to the waveband used in this study. However, comparison with a large sample of nearby $(z<0.11$; De Propris et al. 2002) and intermediateredshift $(z \sim 0.3$; Yagi et al. 2002; Valloto et al. 1997; Gaidos 1997) clusters shows that while $M_{R}^{*}$ values are consistent $\left(M_{R}^{*}=-22.04 \pm 0.11+5 \log h_{65}\right.$ [Gaidos 1997] and $M_{R}^{*}=-22.24 \pm 0.20+5 \log h_{65}$ [Yagi et al. 2002]), our $M_{B}^{*}$ estimate for Coma is fainter by almost 1 mag $\left(M_{B}^{*}=-20.80 \pm 0.10+5 \log h_{65}\right.$ [De Propris et al. 2002; Valloto et al. 1997]). Differences between the faint-end slopes of the LFs in these studies have only a small effect in this comparison. This indicates that galaxies in the Coma are, on average, redder than other clusters. This is unlikely 
to be due to a redshift effect, since the De Propris et al. sample covers a range $z<0.11$. Also, the richness of the Coma Cluster is not responsible for this result since there are many rich clusters in the Yagi et al. sample. Moreover, fitting a subset of their clusters with $\sigma<800 \mathrm{~km} \mathrm{~s}^{-1}$, De Propris et al. still estimate $M_{B}^{*}=-20.72 \pm 0.10+5 \log h_{65}$, similar to that from their full sample. This is likely due to the very large fraction of early-type galaxies in the Coma Cluster, as also shown in Figure 9.

A dip is detected in the total LFs for the Coma Cluster in both the $R$ and $B$ bands. This appears at the same magnitude, scaled by the mean $B-R$ color of galaxies in the Coma. A similar effect is also found in the LFs from other studies of the Coma (Biviano et al. 1995; Driver et al. 1994) and other nearby (Wilson et al. 1997) and intermediateredshift clusters (Yagi et al. 2002; Dahlen et al. 2003), but not in deep-field surveys (Ellis et al. 1996; Cowie et al. 1996). We also find the amplitude of the dip to increase from the $R$ to the $B$ band. The presence of the dip implies a twocomponent shape for the cluster LF, consisting of a Gaussian and a power law at the bright and faint magnitudes, respectively (Biviano et al. 1995; Yagi et al. 2002). Considering the total LF in $B-R$ color intervals in Figure 9, we find a distinct change in the shape of the LF for red galaxies $(B-R>1.35)$ at $M_{R}=-18 \mathrm{mag}$, at both the core (Coma 1) and outskirt (Coma 2 and Coma 3) fields. This result is in qualitative agreement with the finding by Yagi et al. (2002), who attribute the change in the LF shape to contributions from early-type galaxies, defined as those with an $r^{1 / 4}$-law surface brightness profile.

The question of particular interest here concerns the nature of galaxies contributing to the faint end of Coma LF and whether these are different between the Coma 1 and Coma 3 fields. Study of the LF in color intervals (Fig. 9) shows an abundance of low-luminosity red galaxies $(B-R>1.3)$ in Coma 1, compared to star-forming objects $(B-R<1.3)$, with the difference decreasing toward the outskirts (Coma 2 and Coma 3). Moreover, the faint-end slope of the red component of the LF is significantly steeper $(\alpha=-1.6)$ than that observed for blue galaxies $(\alpha=-1.1)$. This implies that low-luminosity red galaxies dominate the faint-end slope of the LF, in agreement with the result in Conselice (2002), who finds a steep faint-end slope for the LF in A0426 due to the presence of a lowmass red population. Therefore, the main contribution to the faint end of the Coma LF (and in clusters studied by De Propris et al. 2002) is the large number of faint red galaxies that are present (presumably produced from the equally large number of faint blue galaxies in the field). A relatively higher surface density for this population in the denser region of the cluster (Coma 1) compared to the less dense region (Coma 3) indicates that dynamical stripping of high-mass systems in cluster environments is, at least partly, responsible for the formation of low-luminosity red galaxies. However, other mechanisms must be at work in less dense regions of the Coma to produce this population. Evidence for gas deficiency in dwarf galaxies in Coma 3 comes from the absence of $\mathrm{H}$ I emission around the N4839 group (Bravo-Alfaro 2000). This indicates that low-luminosity galaxies in the outskirts of the Coma Cluster have gone through a gas-loss process, either through supernova winds (due to their small potential well) or by dynamical effects after passing through the cluster. Indeed, in a study of radial dependence of spectroscopic line indices of these galaxies (after correcting for luminosity dependence), Paper V finds a significant gradient in $\mathrm{Mg}_{2}$, in the sense that galaxies in the core have stronger $\mathrm{Mg}_{2}$ indices and, hence, higher metallicities. This is caused by trapping of the material in galaxies in dense regions due to external pressure by the intracluster medium, leading to higher metallicities. Moreover, X-ray morphology of the N4839 group in the Coma 3 field indicates that this group is in the process of infall, showing evidence of interaction with the cluster (Neumann et al. 2001). Further observational evidence for dynamical stripping in dense regions of clusters comes from the discovery of diffuse arcs at the core of the Coma Cluster (Trentham \& Mobasher 1998; Gregg \& West 1998).

There appears to be a clear separation in luminosity distribution of galaxies as a function of their effective surface brightness. This monotonic surface brightness-luminosity relation is useful in studying properties of dwarf galaxies with respect to giants (Ferguson \& Binggeli 1994). Spectroscopically confirmed members of the Coma Cluster have a relatively higher effective surface brightness $\left(\mu_{\text {eff }}<20 \mathrm{mag}\right.$ $\operatorname{arcsec}^{-2}$ ) compared to field galaxies, implying destruction of low surface brightness galaxies in environments with higher local densities. This is predicted by the harassment scenario of galaxy formation, in which because of a small potential well of low-luminosity/low surface brightness galaxies, up to $90 \%$ of their stellar content could be ejected in dense regions of clusters as a result of interaction with larger galaxies (Moore et al. 1996). However, this same process also predicts formation of low surface brightness galaxies through stripping at the cores of rich clusters (Moore et al. 1999). Therefore, the present data cannot be used to constrain models for the formation of low surface brightness galaxies. High-resolution spectroscopy, measuring features diagnostic of age and metallicity, is found to be more effective in studying formation and evolution of low surface brightness galaxies (Paper V).

\section{SUMMARY}

The spectroscopic survey of galaxies in the Coma Cluster, performed in Paper II, is combined with a brighter sample (Edwards et al. 2002), providing a wide-area $\left(1 \mathrm{deg}^{2}\right)$ and deep $(R \sim 19.5)$ survey, covering both the core and outskirts of the cluster. The final survey consists of a total of 1191 galaxies, of which 760 are spectroscopically confirmed members of the Coma Cluster. After correcting the spectroscopic sample for incompleteness, the LFs are constructed, spanning the range $-23<M_{R}-5 \log h_{65}<-16$ and covering both the core and outskirts of the cluster. Results from this study are summarized as follows:

1. The $R$-band LFs are similar at the core and outskirts of the Coma Cluster, with no evidence of the steep faint-end slope found in previous studies (mostly based on photometric surveys). However, because of spectroscopic limitations, the current sample only extends to $M_{R}=-16$ mag, while the observed upturn in the LF slope is seen at somewhat fainter magnitudes in photometric surveys. The total $R$ band LF for the Coma Cluster, fitted to a Schechter form, is $M_{R}^{*}=-21.79_{-0.09}^{+0.08}+5 \log h_{65}, \alpha=-1.18_{-0.02}^{+0.04}$. This is found to be close to that in the general field.

2. Since the $R$ band mostly measures contributions from the old stellar population, relatively unaffected by star 
formation, the similarity of the LFs implies that the average luminosity for a given galaxy mass is little affected by the processes that dictate their morphologies and spectral types.

3. The total $B$-band LF, fitted to a Schechter form, is $M_{B}^{*}=-19.95_{-0.10}^{+0.15}+5 \log h_{65}, \alpha=-0.96_{-0.02}^{+0.01}$. This shows a dip at $M_{B}^{*}=-18 \mathrm{mag}$. A similar feature, although with smaller amplitude, is observed in the total $R$-band LF at the same luminosity (shifted by mean $B-R$ color). This is likely to be due to contribution from luminous early-type galaxies $\left(M_{B}^{*}<-18 \mathrm{mag}\right)$ to the total LF. This suggests that the total LF could best be fitted by two components, a Gaussian (at bright magnitudes) and a power law (at fainter magnitudes).
4. Study of the LFs in $B-R$ color intervals shows a steep faint-end slope for red $(B-R>1.35)$ galaxies, at both the core and outskirts of the cluster. This population of lowluminosity red galaxies has a higher surface density than the blue $(B-R<1.35)$ star-forming population (at both the core and outskirts of the cluster) and dominates the faint end of the Coma Cluster LF.

5. A monotonic correlation is found between the effective surface brightness and luminosity of galaxies. Cluster galaxies are found to have a higher surface brightness than their field counterparts. This implies destruction of low surface brightness galaxies in dense regions of clusters, indicating the effect of local environment on formation of galaxies.
Adami, C., Ulmer, M. P. Durret, F Nichol, R. C., Mazure, A., Holden, B. P., Romer, A. K., \& Savine, C. 2000, A\&A, 353, 930

Andreon, S., \& Cuillandre, J.-C. 2002, ApJ, 569, 144

Arnaboldi, M., et al. 2002, AJ, 123, 760

Beijersbergen, M., Hoekstra, H., van Dokkum, P. G., \& van der Hulst, T. 2002, MNRAS, 329, 385

Bernstein, G. M., Nichol, R. C., Tyson, J. A., Ulmer, M. P., \& Wittman, D. 1995, AJ, 110, 1507

Biviano, A., Durret, F., Gerbal, D., Le Fèvre, O., Lobo, C., Mazure, A., \& Slezak, E. 1995, A\&AS, 111, 265

Blanton, M. R., et al. 2001, AJ, 121, 2358

Bravo-Alfaro, H., Cayatte, V., van Gorkom, J. H., \& Balkowski, C. 2000, AJ, 119, 580

Brown, W., Geller, M. J., Fabricant, D. G., \& Kurtz, M. J. 2001, AJ, 122, 714

Carter, D., et al. 2002, ApJ, 567, 772 (Paper V)

Chiba, M., \& Nath, B. B. 1994, ApJ, 436, 618

Colless, M., \& Dunn, A. M. 1996, ApJ, 458, 435

Conselice, J. C. 2002, ApJ, 573, L5

Cowie, L. L., Songaila, A., Hu, E \& Cohen, J. G. 1996, AJ, 112, 839

Cross, N., et al. 2001, MNRAS, 324, 825

Dahlen, T., Fransson, C., Ostlin, G., \& Naslund, M. 2003, MNRAS, submitted

Dekel, A., \& Silk, J. 1986, ApJ, 303, 39

De Propris, R., Eisenhardt, P. R., Stanford, S. A., \& Dickinson, M. 1998, ApJ, 503, L45

De Propris, R., et al. 2002, MNRAS, 329, 87

Driver, S. P., Phillips, S., Davies, J. I., Morgan, I., \& Disney, M. J. 1994, MNRAS, 268, 393

Driver, S. P., Windhorst, R. A., \& Griffiths, R. E. 1995, ApJ, 453, 48

Edwards, S. A., Colless, M., Bridges, T. J., Carter, D., Mobasher, B., \& Poggianti, B. M. 2002, ApJ, 567, 178

Efstathiou, G. 1992, MNRAS, 256, 43P

Ellis, R. S., Colless, M., Broadhurst, T. J., Heyl, J., \& Glazebrook, K. 1996, MNRAS, 280, 235

Ferguson, H. C., \& Binggeli, B. 1994, A\&A Rev., 6, 67

Gaidos, E. J. 1997, AJ, 113, 117

Godwin, J. G., Metcalfe, N., \& Peach, J. V. 1983, MNRAS, 202, 113

Gregg, M. D., \& West, M. J. 1998, Nature, 396, 549

Komiyama, Y., et al. 2002, ApJS, 138, 265 (Paper I)

Lobo, C., Biviano, A., Durret, F., Gerbal, D., Le Fèvre, O., Mazure, A., \& Slezak, E. 1997, A\&A, 317, 385

\section{EFERENCES}

López-Cruz, O., Yee, H. K. C., Brown, J. P., Jones, C., \& Forman, W. 1997, ApJ, 475, L97

Mateo, M. L. 1998, ARA\&A, 36, 435

Mobasher, B., \& Trentham, N. 1998, MNRAS, 293, 315

Mobasher, B., et al. 2001, ApJS, 137, 279 (Paper II)

Moore, B., Katz, N., Lake, G., Dressler, A., \& Oemler, A. 1996, Nature, 379,613

Moore, B., Lake, G., Stadel, J., \& Quinn, T. 1999, in IAU Colloq. 171, The Low Surface Brightness Universe, ed. J. I. Davies, C. Impey, \& S. Phillipps (ASP Conf. Ser. 170; San Francisco: ASP), 229

Neumann, D. M., et al. 2001, A\&A, 365, L74

Norberg, P., et al. 2002, MNRAS, 336, 907

Phillipps, S., Driver, S. P., Couch, W. J., \& Smith, R. M. 1998, ApJ, 498, L119

Poggianti, B. M., et al. 2001, ApJ, 562, 689 (Paper III)

Rines, K., Geller, M. J., Kurtz, M. J., Diaferio, A., Jarrett, T. H., \& Huchra, J. P. 2001, ApJ, 561, L41

Schechter, P. L. 1976, ApJ, 203, 297

Secker, J., \& Harris, W. E. 1996, ApJ, 469, 623

Sekiguchi, M., Nakaya, H., Kataza, H., \& Miyazaki, S. 1998, Exp. Astron., 8,51

Somerville, R. S. 2002, ApJ, 572, L23

Sprayberry, D., Impey, C. D., Irwin, M. J., \& Bothun, G. D. 1997, ApJ, 482, 104

Thompson, L. A., \& Gregory, S. A. 1993, AJ, 106, 2197

Thoul, A. A., \& Weinberg, D. H. 1995, ApJ, 442, 480

Trentham, N. 1998, MNRAS, 293, 71

Trentham, N., \& Hodgkins, S. 2002, MNRAS, 333, 423

Trentham, N., \& Mobasher, B. 1998, MNRAS, 293, 53

Trentham, N., Tully, R. B., \& Verheijen, M. A. W. 2001, MNRAS, 325, 385

Tully, R. B., Somerville, R. S., Trentham, N., \& Verheijen, M. A. W. 2002, ApJ, 569, 573

Valotto, C. A., Moore, B., \& Lambas, D. G. 2001, ApJ, 546, 157

Valotto, C. A., Nicotra, M. A., Muriel, H., \& Lambas, D. G. 1997, ApJ, 479,90

West, M., \& Blakeslee, J. P. 2000, ApJ, 543, L27

Wilson, G., Smail, I., Ellis, R. S., \& Couch, W. J. 1997, MNRAS, 284 915

Yagi, M., Kashikawa, N., Sekiguchi, M., Doi, M., Yasuda, N., Shimasaku, K., \& Okamura, S. 2002, AJ, 123, 87

Yasuda, N., Fukugita, M., \& Okamura, S. 1997, ApJS, 108, 417 